\title{
GENERALIZED SOLUTIONS TO HYBRID DYNAMICAL SYSTEMS*
}

\author{
Ricardo G. Sanfelice ${ }^{1}$, Rafal Goebel ${ }^{2}$ and Andrew R. Teel ${ }^{1}$
}

\begin{abstract}
Several recent results in the area of robust asymptotic stability of hybrid systems show that the concept of a generalized solution to a hybrid system is suitable for the analysis and design of hybrid control systems. In this paper, we show that such generalized solutions are exactly the solutions that arise when measurement noise in the system is taken into account.
\end{abstract}

Mathematics Subject Classification. 93C73, 34A37, 93C65

Received May 3, 2006. Revised March 22, 2007.

Published online February 7, 2008.

\section{INTRODUCTION}

\subsection{Contribution}

Hybrid dynamical systems comprise a rich class of systems in which the state can both evolve continuously (flow) and discontinuously (jump). Over the last ten years or more, in research areas such as computer science, feedback control, and dynamical systems, researchers have given considerable attention to modeling and solution definitions for hybrid systems. Some notable references include $[4,8,10,33,44,47,48]$.

In the paper [24], motivated by robust stability issues in hybrid control systems, the authors introduced the notion of a generalized solution to a hybrid system and outlined some stability theory consequences that followed from this solution concept. These included results on "for free" robustness of stability, a generalization of LaSalle's invariance principle, and the existence of smooth Lyapunov functions for asymptotically stable hybrid systems. More details about these results and generalizations were given in the subsequent work [23] and the conference papers [41] and [12], respectively.

The purpose of the current paper is to motivate further the use of generalized hybrid solutions by considering the effect of arbitrary small measurement noise in hybrid control systems. To this end, we show that:

\footnotetext{
Keywords and phrases. Hybrid systems, generalized solutions, differential inclusions, difference inclusions, robust control, hybrid feedback.

* Research partially supported by the Army Research Office under Grant no. DAAD19-03-1-0144, the National Science Foundation under Grant no. CCR-0311084 and Grant no. ECS-0324679, and by the Air Force Office of Scientific Research under Grant no. F49620-03-1-0203. Conference version of this paper entitled "A feedback control motivation for generalized solutions to hybrid systems" appeared without proofs in the Proceedings of the 9th International Workshop Hybrid Systems: Computation and Control, Santa Barbara, 2006.

${ }^{1}$ Center for Control, Dynamical Systems, and Computation; Department of Electrical and Computer Engineering; University of California, Santa Barbara, CA 93106-9560, USA; rsanfelice@ece.ucsb.edu; teel@ece.ucsb.edu

${ }^{2}$ Department of Mathematics, University of Washington, Seattle, WA 98195, USA; goebel@math. washington.edu
} 
For general hybrid systems, including those arising from using hybrid feedback in nonlinear control systems, generalized hybrid solutions agree with the limits (in an appropriate sense) of solutions generated with arbitrarily small state measurement noise.

Our results generalize, to the hybrid setting, a result for differential equations initially reported by Hermes in [26] and expanded upon by Hàjek in [25] and a result by Coron and Rosier [19] given in the context of robust stabilization of nonlinear control systems (with non-hybrid feedback). As a special case, they subsume analogous results for difference equations that, to the best of our knowledge, have not appeared in the literature. While our results appear like a natural generalization of the mentioned work, they are only possible if one from the beginning abandons the standard time as a way to parameterize a solution to a hybrid system and instead uses a generalized notion of "hybrid time", and relies on graphical, rather than uniform or some variation of it, convergence.

\subsection{Initial discussion}

In continuous-time systems, generalized solutions to discontinuous differential equations are shunned at times because using such solutions precludes solving certain nonlinear control problems. For example, for asymptotically controllable nonlinear systems, it is possible to solve the stabilization problem by state feedback when using weak notions of solution for discontinuous differential equations (e.g., Carathéodory solutions, Euler solutions, etc.; see [15]) but it is impossible to solve this problem in general when using generalized solutions such as those due to Krasovskii [30], Filippov [21], or Hermes [26]; for further details see [13].

The feedback stabilization problem does not provide the same motivation for avoiding generalized solutions to hybrid systems. Indeed, it is possible to robustly stabilize asymptotically controllable nonlinear systems using hybrid feedback and using generalized solutions to hybrid systems. See, for example, [38].

Despite our opinion that the use of generalized solutions to hybrid systems will never diminish the capabilities of hybrid control, we would not be surprised to see some resistance to the use of generalized hybrid solutions to hybrid control systems. We expect the main sticking point to be how the notion of generalized solutions affects the "semantics" of a hybrid control system. We now elaborate on what we mean.

For the purposes of this paper, a hybrid system is specified by $f, g, C$, and $D$, which we refer as the data, where $f$ is a function from $C$ to $\mathbb{R}^{n}$ called the "flow map", $g$ is a set-valued mapping from $D$ to $\mathbb{R}^{n}$ called the "jump map", $C \subset \mathbb{R}^{n}$ is called the "flow set" and indicates where in the state space flow may occur, $D \subset \mathbb{R}^{n}$ is called the "jump set" and indicates from where in the state space jumps may occur. For simplicity of the discussion here, we will let $g$ be a function. Then, the hybrid system we study can be expressed in the suggestive form

$$
\mathcal{H}:\left\{\begin{aligned}
\dot{x} & =f(x) & & x \in C \\
x^{+} & =g(x) & & x \in D .
\end{aligned}\right.
$$

The state $x$ can include both the so-called "continuous variables" and the so-called "discrete variables", or modes. Several models for hybrid and switched systems available in the literature (see e.g. $[8,27,34,47]$ ), under certain assumptions, can be fit in such framework. We illustrate this in Section 2; see also [24] for further examples of modeling capabilities of this framework. The particular concepts of a solution to a hybrid system we use in this paper will be made precise in Section 2. Such concepts are not relevant for the discussion below.

Generalized solutions to $\mathcal{H}$ are solutions to a hybrid system with regularized data $\widehat{f}, \widehat{g}, \widehat{C}, \widehat{D}$, where $\widehat{f}$ and $\widehat{g}$ are constructed from $f$ and $g$ in a manner that will be made precise later (see Def. 2.12) and $\widehat{C}$ and $\widehat{D}$ denote the closures of $C$ and $D$ (relative to an open state space that we specify later). In particular, for systems on $\mathbb{R}^{n}$, this means that if $C \cup D=\mathbb{R}^{n}$ then $\widehat{C} \cap \widehat{D}=\bar{C} \cap \bar{D}$ is not empty ${ }^{1}$ even if $C \cap D$ is empty. It turns out that many models of hybrid systems insist on having $C \cap D$ empty; see for example [14]. In fact, one has $C=\mathbb{R}^{n} \backslash D$ in the impulsive dynamical systems considered in $[3,5,6]$. The condition $C=\mathbb{R}^{n} \backslash D$ is also used in many of the hybrid models considered in [8]. In simulations of hybrid systems, the set $D$ is usually specified in the simulator while the the set $C$ is not, which is usually automatically taken by the simulator to be $\mathbb{R}^{n} \backslash D$. Making $C \cap D$

\footnotetext{
${ }^{1}$ This is true unless either $C$ is empty or $D$ is empty, in which case the original system was not truly hybrid in the first place.
} 
empty is one way to guarantee that jumps are enforced in the jump set rather than simply enabled. (Some researchers use the phrases "as is' semantics" and "enabling semantics" for these two respective situations, see [42].) Moreover, it is a way to guarantee that solutions, if they exist, are unique when the flow map $f$ is locally Lipschitz.

As we pointed out in [24], changing $C$ and $D$ to their relative closures can have a dramatic effect on the solutions to the hybrid control system. For example, if $D$ has measure zero, perhaps being a surface on which jumps are enforced, and $C=\mathbb{R}^{n} \backslash D$ (see, for example, the model of reset control systems used in [6] and the references therein) then the relative closure of $C$ will be equal to the entire state space $\mathbb{R}^{n}$. This may enable solutions that never jump ${ }^{2}$, circumventing the controller's hybrid nature. However, the point we are making in this paper is that the new behavior that appears when taking the relative closures can manifest itself due to measurement noise in a feedback control system. In this sense, this new behavior should be taken into account.

There are many motivations for not taking the flow set $C$ and the jump set $D$ to be closed sets when defining the data of a hybrid system. However, in the context of hybrid control systems, we hope that the robust stability motivation given in [24], the solution properties reported in [23], the stability theory corollaries reported in [23] and [41], and the new results reported here on the equivalence between generalized solutions and the limit of solutions due to measurement noise continue to motivate the development of hybrid control system models that use jump and flow sets that are closed relative to the state space. An example in this direction is the work of [36,49] which revisits the reset control systems considered in [6] and finds a natural definition of the flow set and jump set so that they are closed and yet still force jumps at the appropriate locations in the state space.

\section{Definitions of Generalized SOlutions}

In what follows we write $\mathbb{R}_{\geq 0}$ for $[0, \infty), \mathbb{N}$ for $\{0,1,2, \ldots\}, \mathbb{B}$ for the closed unit ball in $\mathbb{R}^{n}$, and $|\cdot|$ for the Euclidean vector norm.

\subsection{Data of hybrid systems}

In what follows, we will describe two concepts of generalized solutions to hybrid systems of the type

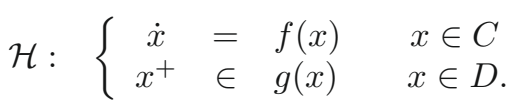

Above, $f: C \rightarrow \mathbb{R}^{n}$ is the "flow map", $C \subset \mathbb{R}^{n}$ is the "flow set", $g: D \rightrightarrows \mathbb{R}^{n}$ is the "jump map" (the doublearrow, here and in what follows, indicates a set-valued mapping) and $D \subset \mathbb{R}^{n}$ is the "jump set". All of the interesting issues we address in this paper arise even if $g$ is just a (single-valued) function. We allow $g$ to be a set-valued mapping to allow for some of the systems that arise in the study of hybrid automata.

Hybrid systems as in (1), with appropriate choice of data, can model systems where the "discrete variable" $q$ is explicitly mentioned. More specifically, consider

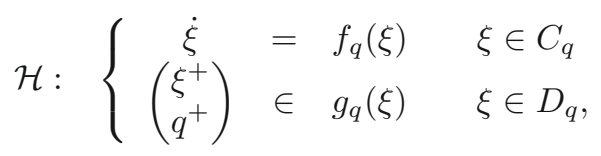

where $Q$ is the set of "modes", and for each $q \in Q, f_{q}: C_{q} \rightarrow \mathbb{R}^{m}, C_{q} \subset \mathbb{R}^{m}, g_{q}: D_{q} \rightrightarrows \mathbb{R}^{m} \times Q$, and $D_{q} \subset \mathbb{R}^{m}$. When $Q$ can be identified with a subset of integers (and so a subset of $\mathbb{R}$ ), we can consider a system

\footnotetext{
${ }^{2}$ Just because $D$ has measure zero does not automatically imply that generalized solutions will include solutions that never jump. For example, in the bouncing ball system in [28], where $h$ denotes the height of the ball, the jump and flow sets are given by $D=\left\{(h, \dot{h}) \in \mathbb{R}^{2} \mid h \leq 0, \dot{h}<0\right\}, C=\left\{(h, \dot{h}) \in \mathbb{R}^{2} \mid h>0\right\}$, respectively. Note that $C \neq \mathbb{R}^{2} \backslash D$ here. The only extra solution to the bouncing ball introduced by generalized solutions is an instantaneous Zeno solution at the origin.
} 
in the form (1) with the variable $x=\left(\begin{array}{l}\xi \\ q\end{array}\right) \in \mathbb{R}^{m+1}$ and data

$$
f(x)=\left(\begin{array}{c}
f_{q}(\xi) \\
0
\end{array}\right), \quad C=\bigcup_{q \in Q}\left(C_{q} \times\{q\}\right), g(x)=g_{q}(\xi), \quad D=\bigcup_{q \in Q}\left(D_{q} \times\{q\}\right) .
$$

In turn, systems in the form (2) easily capture the commonly encountered hybrid systems (hybrid automata) with data given in terms of domains, guards, edges, and resets, as found for example in $[9,11,34]$. Let $Q$ be the set of "modes", for each $q \in Q$ let $f_{q}$ be the flow map, let the mapping Domain : $Q \rightrightarrows \mathbb{R}^{m}$ give the domains of flow, let Edges $\subset Q \times Q$ be the set of edges, Guard : Edges $\rightrightarrows \mathbb{R}^{m}$ be the mapping giving the guards, and Reset : Edges $\times \mathbb{R}^{m} \rightrightarrows \mathbb{R}^{m}$ be the reset map. One then takes

$$
C_{q}=\operatorname{Domain}(q), \quad D_{q}=\bigcup_{e=\left(q, q^{\prime}\right) \in \operatorname{Edges}} \operatorname{Guard}(e), g_{q}(x)=\bigcup_{\substack{e=\left(q, q^{\prime}\right) \text { s.t. } \\
x \in \operatorname{Guard}(e)}}\left(\begin{array}{c}
\operatorname{Reset}(e, x) \\
q^{\prime}
\end{array}\right) .
$$

Note that a set-valued mapping $g_{q}$ will arise this way when two guards sets, Guard $\left(e^{\prime}\right)$ with $e^{\prime}=\left(q, q^{\prime}\right)$ and $\operatorname{Guard}\left(e^{\prime \prime}\right)$ with $e^{\prime \prime}=\left(q, q^{\prime \prime}\right)$, overlap. This will even be the case when the resets $\operatorname{Reset}\left(e^{\prime}, \cdot\right)$ and $\operatorname{Reset}\left(e^{\prime \prime}, \cdot\right)$ are single valued.

Example 2.1. Consider the system in [48], Example 1, which consists of two relays whose state is either open or closed and that are controlled by the values of continuous states $\xi_{1}, \xi_{2} \in \mathbb{R}$, respectively. Having $\xi_{1}=0$ forces the closing of the first relay, $\xi_{2}=0$ forces the closing of the second relay. Once the relays are closed, they are latched, regardless of the value of $\xi_{i}$ 's. Let $\xi:=\left[\begin{array}{ll}\xi_{1} & \xi_{2}\end{array}\right]^{T}$. Four different modes $q \in Q:=\{1,2,3,4\}$ describe the state of the relays: $q=1$ indicates when both relays are open, $q=2$ indicates when only the first relay is closed, $q=3$ indicates when only the second relay is closed, $q=4$ indicates when both relays are closed. Only the transitions from $1 \rightarrow 2,1 \rightarrow 3,1 \rightarrow 4,2 \rightarrow 4$, and $3 \rightarrow 4$ are modeled. The control logic for the activation of the relays can be written as a hybrid automaton with modes $q \in\{1,2,3,4\}$, flow maps that we assume to be given by $f_{q}(\xi)=\left(\begin{array}{l}1 \\ 1\end{array}\right)$ for all $q \in Q$, domains

$$
\begin{aligned}
& \operatorname{Domain}(1):=\left\{\xi \in \mathbb{R}^{2} \mid \xi_{1} \leq 0, \xi_{2} \leq 0\right\}, \quad \operatorname{Domain}(2):=\left\{\xi \in \mathbb{R}^{2} \mid \xi_{1} \geq 0, \xi_{2} \leq 0\right\}, \\
& \operatorname{Domain}(3):=\left\{\xi \in \mathbb{R}^{2} \mid \xi_{1} \leq 0, \xi_{2} \geq 0\right\}, \quad \operatorname{Domain}(4):=\left\{\xi \in \mathbb{R}^{2} \mid \xi_{1} \geq 0, \xi_{2} \geq 0\right\},
\end{aligned}
$$

set of edges Edges $=\{(1,2),(1,3),(1,4),(2,4),(3,4)\}$, guard sets

$$
\begin{aligned}
& \operatorname{Guard}((1,2)):=\left\{\xi \in \mathbb{R}^{2} \mid \xi_{1}=0, \xi_{2}<0\right\}, \operatorname{Guard}((1,3)):=\left\{\xi \in \mathbb{R}^{2} \mid \xi_{1}<0, \xi_{2}=0\right\}, \\
& \operatorname{Guard}((1,4)):=\left\{\xi \in \mathbb{R}^{2} \mid \xi_{1}=0, \xi_{2}=0\right\}, \operatorname{Guard}((2,4)):=\left\{\xi \in \mathbb{R}^{2} \mid \xi_{1} \geq 0, \xi_{2}=0\right\} \\
& \operatorname{Guard}((3,4)):=\left\{\xi \in \mathbb{R}^{2} \mid \xi_{1}=0, \xi_{2} \geq 0\right\}
\end{aligned}
$$

and the reset map $\operatorname{Reset}\left(\left(q, q^{\prime}\right), \xi\right):=\xi$. The closed-loop system can be written as a hybrid system $\mathcal{H}$ as in (2) with state $x=\left(\begin{array}{l}\xi \\ q\end{array}\right)$, flow maps $f_{q}$, flow sets $C_{q}=\operatorname{Domain}(q)$, jump sets

$$
D_{1}=\operatorname{Guard}((1,2)) \cup \operatorname{Guard}((1,3)) \cup \operatorname{Guard}((1,4)), \quad D_{2}=\operatorname{Guard}((2,4)), \quad D_{3}=\operatorname{Guard}((3,4)), \quad D_{4}=\emptyset,
$$


and jump maps

$$
\begin{aligned}
& g_{1}(\xi)=\left(\begin{array}{l}
\xi \\
q
\end{array}\right) \quad \text { if } \quad \xi \in \operatorname{Guard}((1, q)), \quad q=2,3,4 \\
& g_{2}(\xi)=\left(\begin{array}{l}
\xi \\
4
\end{array}\right) \quad \text { if } \quad \xi \in \operatorname{Guard}((2,4)), \\
& g_{3}(\xi)=\left(\begin{array}{l}
\xi \\
4
\end{array}\right) \quad \text { if } \quad \xi \in \operatorname{Guard}((3,4)) .
\end{aligned}
$$

Then, $f, g, C$, and $D$ are given as in (3). Note that $g$ is set valued.

\subsection{Generalized time domain and hybrid arcs}

Independently of how the data of a hybrid system is described, one can give different meanings to a concept of a solution. In what could be described as a "classical" approach to dynamical systems with jumps, the candidates for a solution are considered to be piecewise continuous functions of time that are right continuous and have left limits at each $t$ in their domain of definition. This approach has been used in the early work by Witsenhausen [48] and Tavernini [44], and more recently in [5,14,22,27,28,35,45]. (Functions that are right continuous and have left limits are frequently referred to in the literature as CADLAG, from the French "continue à droite, limite à gauche".)

A $C A D L A G$ solution to $\mathcal{H}=\left(f, g, C, D, \mathbb{R}^{n}\right)$ is a function $\xi:[0, T] \rightarrow \mathbb{R}^{n}$ or $\xi:[0, T) \rightarrow \mathbb{R}^{n}$ that is piecewise absolutely continuous (or piecewise differentiable), has a finite number of discontinuities in each compact subset of the interval on which it is defined, and that at every $t$ it has left limits, is right continuous, and:

(C1) on each interval of continuity

$$
\xi(t) \in C, \quad \dot{\xi}(t)=f(\xi(t))
$$

(C2) at all points $\tau>0$ of discontinuity satisfies

$$
\xi^{-}(\tau) \in D, \quad \xi(\tau) \in g\left(\xi^{-}(\tau)\right)
$$

where $\xi^{-}(\tau):=\lim _{t \nearrow \tau} \xi(t)$. Whether $\dot{\xi}(t)=f(\xi(t))$ is to be understood as true for all $t$ or for almost all $t$ depends on whether one considers $\xi$ 's that are differentiable on the intervals of continuity, or just absolutely continuous. By design, such concept of a solution excludes multiple jumps at a single time instant. Furthermore, it makes it troublesome (or impossible) to discuss limits of solutions; see Example 2.4. These issues can be overcome by using a "generalized" time domain, as defined below.

Definition 2.2 (hybrid time domain). A subset $E \subset \mathbb{R}_{\geq 0} \times \mathbb{N}$ is a compact hybrid time domain if

$$
E=\bigcup_{j=0}^{J-1}\left(\left[t_{j}, t_{j+1}\right] \times\{j\}\right)
$$

for some finite sequence of times $0=t_{0} \leq t_{1} \leq t_{2} \leq \ldots \leq t_{J}$. It is a hybrid time domain if for all $(T, J) \in E$, $E \cap([0, T] \times\{0,1, \ldots, J\})$ is a compact hybrid domain.

Hybrid time domains are similar to hybrid time trajectories in [3,33,34], and to the concept of time evolution in [47], but give a more prominent role to the number of jumps $j$ ( $c f$. the definition of hybrid time set by Collins in [17]). On each hybrid time domain there is a natural ordering of points: we write $(t, j) \preceq\left(t^{\prime}, j^{\prime}\right)$ for $(t, j),\left(t^{\prime}, j^{\prime}\right) \in E$ if $t \leq t^{\prime}$ and $j \leq j^{\prime}$.

Definition 2.3 (hybrid arc). A hybrid arc is a function $x: \operatorname{dom} x \rightarrow \mathbb{R}^{n}$, where dom $x$ is a hybrid time domain, that is locally absolutely continuous in $t$ on $\operatorname{dom} x \cap\left(\mathbb{R}_{\geq 0} \times\{j\}\right)$ for each $j \in \mathbb{N}$. 


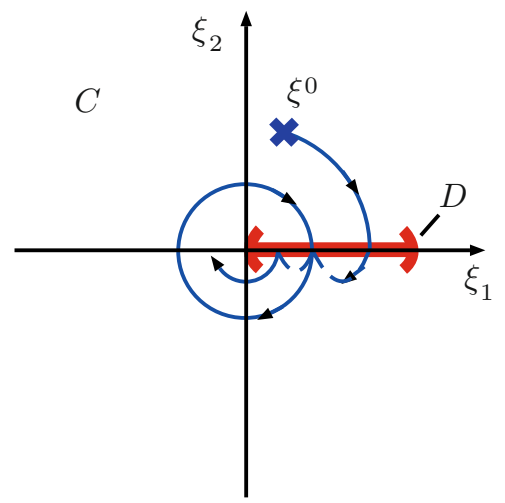

Figure 1. A CADLAG solution $\xi$ to $\mathcal{H}$ in Example 2.4 starting from $x^{0}$. When it reaches the $D$ set, its magnitude is reduced by half. Flows continue after the jump until the solution hits the $D$ set again.

Hybrid $\operatorname{arcs} x$ are parameterized by $(t, j) \in \operatorname{dom} x$, with $x(t, j)$ being the value of $x$ at the "hybrid instant" given by $(t, j)$. A hybrid arc $x$ is said to be nontrivial if dom $x$ contains at least one point different from $(0,0)$, complete if $\operatorname{dom} x$ is unbounded, Zeno if it is complete but the projection of $\operatorname{dom} x$ onto $\mathbb{R}_{\geq 0}$ is bounded, and instantaneously Zeno if $\operatorname{dom} x=\{0\} \times \mathbb{N}$.

We note that with each CADLAG solution, one can naturally associate a hybrid arc. More specifically, if $t_{j}$, $j=1,2, \ldots, J$, is the time of the $j$-th jump of a CADLAG solution $\xi:[0, T] \rightarrow \mathbb{R}^{n}$, and $t_{0}=0, t_{J+1}=T$, one can associate with $\xi$ a hybrid arc $\tilde{\xi}$ on a hybrid time domain given by $\bigcup_{j=1}^{J}\left(\left[t_{j}, t_{j+1}\right] \times\{j\}\right)$, with $\tilde{\xi}(t, j)=\xi(t)$ for all $t \in\left[t_{j}, t_{j+1}\right), \tilde{\xi}\left(t_{j+1}, j\right)=\xi^{-}\left(t_{j+1}\right)$ (recall that $\xi^{-}\left(t_{j+1}\right)=\lim _{t} t_{j+1} \xi(t)$ ). Then $\tilde{\xi}$ is an execution of $\mathcal{H}$ (another commonly used concept of a solution to hybrid systems; see for example [34]), in the sense that:

(E1) $\tilde{\xi}(t, j) \in C$ for all $t \in\left[t_{j}, t_{j+1}\right)$ while $\dot{\tilde{\xi}}(t, j)=f(\tilde{\xi}(t, j))$ for almost all $t \in\left[t_{j}, t_{j+1}\right)$ (or for all $t \in\left[t_{j}, t_{j+1}\right)$ if the original solution was piecewise differentiable);

(E2) $\tilde{\xi}\left(t_{j+1}, j\right) \in D, \tilde{\xi}\left(t_{j+1}, j+1\right) \in g\left(\tilde{\xi}\left(t_{j+1}, j\right)\right)$, for $j=0,1, \ldots, J$.

The following example illustrates that, compared to using CADLAG functions, hybrid arcs (and the concept of an execution) can lead to a larger set of solutions.

Example 2.4. Consider a hybrid system $\mathcal{H}$ on $\mathbb{R}^{2}$ given by

$$
\dot{x}=f(x):=\left(\begin{array}{c}
x_{2} \\
-x_{1}
\end{array}\right) \quad x \in C:=\mathbb{R}^{2}, \quad x^{+}=g(x):=\frac{x}{2} \quad x \in D:=(0,1) \times\{0\} .
$$

For any point $x^{0}$ with $0<\left|x^{0}\right|<1$ and $x^{0} \notin D$, a CADLAG solution from $x^{0}$ exists. One such solution rotates clockwise around the origin for all time. (Such a solution will be excluded if $D$ is considered to "force" jumps rather than "enable" them.) Another solution rotates clockwise until it hits $D$, then via a jump has its magnitude divided by 2 , and then rotates again for $2 \pi$ units of time until it hits $D$ again at which point in time it jumps, and this cycle repeats for all time. Figure 1 depicts a solution of this type up to the second jump. Other solutions are created by rotating clockwise around the origin when not in $D$ and permitting a jump whenever in $D$.

There are additional solutions that are admitted when using hybrid time domains that are not admitted when using CADLAG functions. In particular, the hybrid arc that corresponds to rotating clockwise around the origin until hitting $D$ and then making an infinite number of jumps, each one cutting the magnitude of the solution in half, is a solution since each jump maps a point in $D$ to another point in $D$. 


\subsection{Local boundedness assumptions and a key definition: graphical convergence}

To obtain meaningful concepts of generalized solutions, we will need to restrict our attention to an open set $O \subset \mathbb{R}^{n}$ on which $f$ and $g$ have reasonable local boundedness properties.

In general, given an open set $O$, a function $\phi: S \rightarrow \mathbb{R}^{n}$ (or a set-valued mapping $\phi: S \rightrightarrows \mathbb{R}^{n}$ ) defined on a subset $S \subset \mathbb{R}^{n}$ is locally bounded on $O$ if for each compact set $K \subset O$ there exists a compact set $K^{\prime} \subset \mathbb{R}^{n}$ such that $\phi(K) \subset K^{\prime}$. It is locally bounded with respect to $O$ on $O$ if we request that $K^{\prime} \subset O$.

Assumption 2.5. The set $O \subset \mathbb{R}^{n}$ is open. The sets $C$ and $D$ are subsets of $O$. The function $f: C \rightarrow \mathbb{R}^{n}$ is locally bounded on $O$. The set-valued mapping $g: D \rightrightarrows \mathbb{R}^{n}$ is locally bounded with respect to $O$ on $O$.

Essentially, this means that solutions to $\mathcal{H}$ (in the CADLAG or execution sense, or even in the generalized sense we define later) have some uniform continuity properties when flowing in a compact subset of $O$ and do not jump from that compact set to locations arbitrarily close to the boundary of $O$.

To define solutions to a hybrid system with measurement noise, we will need a concept of convergence of hybrid arcs that admits sequences of arcs with potentially different domains. To this end, we will rely on graphical convergence. Given a hybrid $\operatorname{arc} x: \operatorname{dom} x \rightarrow \mathbb{R}^{n}$, its graph is the set

$$
\operatorname{gph} x:=\left\{(t, j, x(t, j)) \in \mathbb{R}_{\geq 0} \times \mathbb{N} \times \mathbb{R}^{n} \mid(t, j) \in \operatorname{dom} x\right\}
$$

A sequence of hybrid $\operatorname{arcs}\left\{x_{i}\right\}_{i=1}^{\infty}$ converges graphically to a hybrid arc $x$ if the sequence of graphs $\left\{\text { gph } x_{i}\right\}_{i=1}^{\infty}$ converges to gph $x$ in the sense of set convergence. The latter concept is well-established and often used in set-valued and nonsmooth analysis; see $[2,39]$. For precise definitions of general set and graphical convergence we refer the reader to [39], Chapters 4 and 5 (below we state a version of [39], Exercise 5.34, relevant for our purposes). For further details on graphical convergence of hybrid arcs we recommend [23]. Finally, we add that graphical convergence is closely related to convergence in the Skorokhod topology used in [17].

Lemma 2.6 (graphical convergence of hybrid arcs). Let $x$ be a hybrid arc with compact dom $x$, and let $(T, J)$ be the supremum of $\operatorname{dom} x$. A sequence $\left\{x_{i}\right\}_{i=1}^{\infty}$ of hybrid arcs with $\operatorname{dom} x_{i} \subset \mathbb{R}_{\geq 0} \times\{0,1, \ldots, J\}, i=1,2, \ldots$, converges graphically to $x$ if and only if for all $\varepsilon>0$, there exists $i_{0} \in \mathbb{N}$ such that, for all $i>i_{0}$

(a) for all $(t, j) \in \operatorname{dom} x$ there exists s such that $(s, j) \in \operatorname{dom} x_{i},|t-s|<\varepsilon$, and $\left|x(t, j)-x_{i}(s, j)\right|<\varepsilon$,

(b) for all $(t, j) \in \operatorname{dom} x_{i}$ there exists s such that $(s, j) \in \operatorname{dom} x,|t-s|<\varepsilon$, and $\left|x_{i}(t, j)-x(s, j)\right|<\varepsilon$.

In particular, a sequence $\left\{x_{i}\right\}_{i=1}^{\infty}$ of hybrid arcs with $\operatorname{dom} x_{i} \subset \operatorname{dom} x, i=1,2, \ldots$, converges graphically to $x$ if for all $\varepsilon>0$ there exists $i_{0} \in \mathbb{N}$ such that, for all $i>i_{0}$, all $(t, j) \in \operatorname{dom} x$, we have $(t, j) \in \operatorname{dom} x_{i}$ and $\left|x(t, j)-x_{i}(t, j)\right|<\varepsilon$.

\subsection{Generalized solutions à la Hermes}

Equipped with graphical convergence, we generalize the definition of Hermes solutions discussed by Hermes in [26] and later defined by Hàjek in [25]. This corresponds to a concept of solutions to hybrid systems with measurement noise.

Definition 2.7 (hybrid Hermes solution to $\mathcal{H}$ ). A hybrid $\operatorname{arc} \varphi: \operatorname{dom} \varphi \rightarrow O$ is a hybrid Hermes solution to $\mathcal{H}=(f, g, C, D, O)$ if for each compact hybrid time domain $E \subset \operatorname{dom} \varphi$ with the supremum $(T, J)$ and the truncation $\varphi^{E}$ of $\varphi$ to $E$, there exists a sequence of hybrid $\operatorname{arcs} \varphi_{i}: \operatorname{dom} \varphi_{i} \rightarrow O$ and measurable functions $e_{i}$ : $\operatorname{dom} e_{i} \rightarrow \mathbb{R}^{n}$ with domains $\operatorname{dom} e_{i}=\operatorname{dom} \varphi_{i} \subset \mathbb{R}_{\geq 0} \times\{0,1, \ldots, J\}$, that satisfy: for each $i$, with $\operatorname{dom} \varphi_{i}$ expressed as $\bigcup_{j=0}^{J}\left(\left[t_{j}^{i}, t_{j+1}^{i}\right] \times\{j\}\right)$,

(H1) for all $j=0,1, \ldots, J-1$,

$$
\varphi_{i}(t, j)+e_{i}(t, j) \in C \text { for all } t \in\left[t_{j}^{i}, t_{j+1}^{i}\right), \quad \dot{\varphi}_{i}(t, j)=f\left(\varphi_{i}(t, j)+e_{i}(t, j)\right) \text { for almost all } t \in\left[t_{j}^{i}, t_{j+1}^{i}\right]
$$


(H2) for all $j=0,1, \ldots, J-1$,

$$
\varphi_{i}\left(t_{j+1}^{i}, j\right)+e_{i}\left(t_{j+1}^{i}, j\right) \in D, \quad \varphi_{i}\left(t_{j+1}^{i}, j+1\right) \in g\left(\varphi_{i}\left(t_{j+1}^{i}, j\right)+e_{i}\left(t_{j+1}^{i}, j\right)\right)
$$

and $\lim _{i \rightarrow \infty} \varphi_{i}(0,0)=\varphi(0,0),\left\{\varphi_{i}\right\}_{i=0}^{\infty}$ converges graphically to $\varphi^{E}$, for each $i$ we have $\sup _{(t, j) \in \operatorname{dom} e_{i}}\left|e_{i}(t, j)\right|=$ : $\varepsilon_{i}<\infty$, and the sequence $\left\{\varepsilon_{i}\right\}_{i=0}^{\infty}$ converges to 0 .

Note that in (H1) we do not insist that $\varphi_{i}\left(t_{j+1}^{i}, j\right)+e_{i}\left(t_{j+1}^{i}, j\right) \in C$. In all systems where $C \cap D=\emptyset$, such condition would exclude solutions that jump after flowing.

Even if a hybrid system is reduced to a differential equation with a constraint, and uniform convergence can replace graphical convergence, Hermes solutions differ significantly from classical ones.

Example 2.8. On $\mathbb{R}^{2}$, consider a hybrid system with $f(x)=\left(\begin{array}{l}1 \\ 1\end{array}\right)$ if $x_{1}$ is rational and $f(x)=\left(\begin{array}{c}1 \\ -1\end{array}\right)$ if $x_{1}$ is irrational, and $C=[0, \infty) \times\{0\}$. There are no absolutely continuous $\operatorname{arcs} \xi$ with $\dot{\xi}(t)=f(\xi(t))$ and $\xi(t) \in C$ for almost all $t$ in a nontrivial time interval - any arc with $\xi(t) \in C$ must satisfy $\dot{\xi}_{2}(t)=0$ for all $t$. However, for any $\varepsilon>0$ there exists a "seesaw" arc $\xi_{\varepsilon}$ and error $e$ on $[0, \infty)$ such that $\xi_{\varepsilon}(t)+e(t) \in C$ and $\dot{\xi}(t)=f(\xi(t)+e(t))$, while $\xi_{\varepsilon}(0)=(0,0),\left|\xi_{\varepsilon}(t)\right| \leq \varepsilon$ and $|e(t)| \leq \varepsilon$. As $\varepsilon \searrow 0$, arcs $\xi_{\varepsilon}$ converge graphically (and uniformly) to the arc $t \mapsto(t, 0)$. Such arc is a Hermes solution to the system.

To illustrate what graphical convergence (vs. classical convergence notions) grants us for general hybrid systems, we give the following, somewhat extreme, example. It will also illustrate that CADLAG concept of a solution does not behave well under graphical convergence, and when executions are considered, graphical convergence may "expose" flaws in the data of a system.

Example 2.9. Consider the system $\mathcal{H}$ from Example 2.4. Let $x$ be a CADLAG solution from $x^{0}$ with $0<\left|x^{0}\right|<1$ and $x^{0} \notin D$. Let $\tau$ be the first time when $x(\tau) \in D$ and consider the noise $e(t)=(0,0)$ if $t \leq \tau, e(t)=(0, \varepsilon)$ for $t>\tau$. Then, one CADLAG solution $\xi$ to $\dot{x}=f(x+e), x+e \in C$ with jumps governed by $x^{+}=g\left(x^{-}+e^{-}\right), x^{-}+e^{-} \in D$ will jump at $\tau$ and then again when $\xi_{2}=-\varepsilon$ since by definition of $e$, after the first jump at $\tau$ there exists $\tau^{\prime}>\tau$ at which $\xi\left(\tau^{\prime}\right)+e\left(\tau^{\prime}\right) \in D$. This way, one can generate sequences of hybrid $\operatorname{arcs} \xi_{i}$ from $x^{0}$ and noise signals $e_{i}$ satisfying (H1), (H2) above, such that $\xi_{i}$ that rotates to $D$, and then jumps infinitely many times, with jumps separated by less than $1 / i$ amount of time. The graphical limit $\xi$ of such $\xi_{i}$ 's flows to $D$ and then jumps infinitely many times. Figure 2 illustrates this limiting process. Of course, such $\xi$ does not correspond to a CADLAG solution. It is an execution, though. However, if one considers a sequence of $\xi_{i}$ 's as above, but with initial points on the line $x_{1}=x_{2}$ and converging to $(0,0)$, the graphical limit is instantaneous Zeno: it jumps infinitely many times from $(0,0)$ to $(0,0)$ with no intervals of flow. While such limit is a hybrid arc, it is not an execution of $\mathcal{H}$ since $(0,0) \notin D$. By definition, it is a Hermes solution.

In many control applications, the state of the system cannot be measured exactly since it is corrupted by noise. The measurement noise can appear in some but not every component of the state (e.g. when state feedback is implemented, noise appears only on states measured with specific sensors). To account for such cases, we consider functions $f$ and $g$ given as

$$
\forall x \in C \quad f(x):=f^{\prime}\left(x, \kappa_{c}(x)\right), \quad \forall x \in D \quad g(x):=g^{\prime}\left(x, \kappa_{d}(x)\right) .
$$

Alternatively, hybrid systems with such structure and with $g(x)=\kappa_{d}(x)$ arise in robust hybrid feedback stabilization of general nonlinear (and not hybrid) control systems; see [37,38].

We will allow for $\kappa_{c}, \kappa_{d}$ to be discontinuous. More specifically, for this case we will assume:

Assumption 2.10. The set $O \subset \mathbb{R}^{n}$ is open. The sets $C$ and $D$ are subsets of $O$. The function $f^{\prime}: O \times$ $\mathbb{R}^{m_{c}} \rightarrow \mathbb{R}^{n}$ is locally Lipschitz continuous in the first argument, locally uniformly in the second argument ${ }^{3}$.

\footnotetext{
${ }^{3}$ A function $h: O \times \mathbb{R}^{m} \rightarrow \mathbb{R}^{n}$ is continuous (locally Lipschitz continuous) in the first argument, locally uniformly in the second argument if for each $z \in O$, each compact $U \subset \mathbb{R}^{m}$, and each $\varepsilon>0$ there exists $\delta>0(K>0)$ such that $|x-z|<\delta$ implies $|h(x, u)-h(z, u)|<\varepsilon(x, y \in(z+\varepsilon \mathbb{B}) \cap O$ implies $|h(x, u)-h(y, u)| \leq K|x-y|)$ for all $u \in U$.
} 


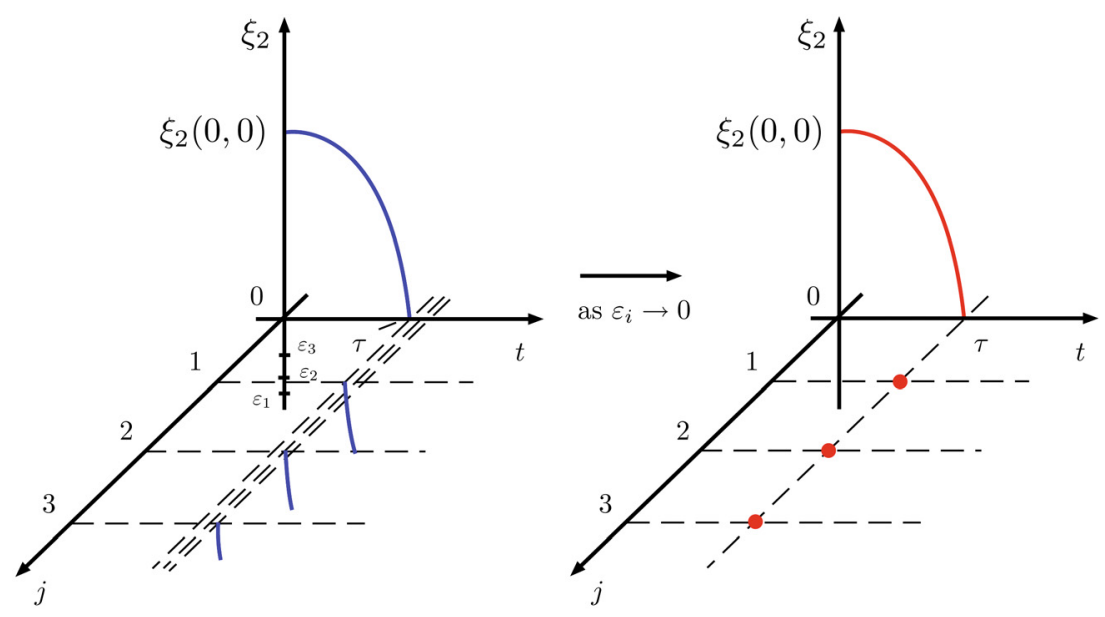

Figure 2. Convergence of solutions to the hybrid system in Example 2.9 when the noise approaches zero. The value of the noise $e$ at the $i$-th jump is given by $\left(0, \varepsilon_{i}\right)$ where $\varepsilon_{i} \rightarrow 0$ as $i \rightarrow \infty$. The limiting function is not a CADLAG solution.

The function $g^{\prime}: O \times \mathbb{R}^{m_{d}} \rightarrow O$ is continuous in the first argument, locally uniformly in the second argument. The functions $\kappa_{c}: C \rightarrow \mathbb{R}^{m_{c}}, \kappa_{d}: D \rightarrow \mathbb{R}^{m_{d}}$ are locally bounded on $O$.

In particular, this assumption guarantees that $f$ and $g$ as defined in (5) satisfy Assumption 2.5.

The notion of Hermes solution in Definition 2.7 changes for a hybrid system $\mathcal{H}=(f, g, C, D, O)$ with $f$ and $g$ given by (5) since the noise is affecting the differential and difference equations only through the function $\kappa_{c}$ and $\kappa_{d}$.

Definition 2.11 (hybrid control-Hermes solution to $\mathcal{H}$ ). A hybrid $\operatorname{arc} \varphi: \operatorname{dom} \varphi \rightarrow O$ is a hybrid control-Hermes solution to $\mathcal{H}=(f, g, C, D, O)$ with $f$ and $g$ given in (5) if for each compact hybrid time $\operatorname{domain} E \subset \operatorname{dom} \varphi$ with supremum $(T, J)$ and the truncation $\varphi^{E}$ of $\varphi$ to $E$, there exists a sequence of hybrid $\operatorname{arcs} \varphi_{i}: \operatorname{dom} \varphi_{i} \rightarrow O$ and measurable functions $e_{i}: \operatorname{dom} e_{i} \rightarrow \mathbb{R}^{n}$, with $\operatorname{dom} e_{i}=\operatorname{dom} \varphi_{i} \subset \mathbb{R}_{\geq 0} \times\{0,1, \ldots, J\}$, that satisfy: for each $i$, with dom $\varphi_{i}$ expressed as $\bigcup_{j=0}^{J}\left(\left[t_{j}^{i}, t_{j+1}^{i}\right] \times\{j\}\right)$,

(cH1) for all $j=0,1, \ldots, J-1$,

$\varphi_{i}(t, j)+e_{i}(t, j) \in C$ for all $t \in\left[t_{j}^{i}, t_{j+1}^{i}\right), \quad \dot{\varphi}_{i}(t, j)=f^{\prime}\left(\varphi_{i}(t, j), \kappa_{c}\left(\varphi_{i}(t, j)+e_{i}(t, j)\right)\right)$ for almost all $t \in\left[t_{j}^{i}, t_{j+1}^{i}\right]$;

(cH2) for all $j=0,1, \ldots, J-1$,

$$
\varphi_{i}\left(t_{j+1}^{i}, j\right)+e_{i}\left(t_{j+1}^{i}, j\right) \in D, \quad \varphi_{i}\left(t_{j+1}^{i}, j+1\right)=g^{\prime}\left(\varphi_{i}\left(t_{j+1}^{i}, j\right), \kappa_{d}\left(\varphi_{i}\left(t_{j+1}^{i}, j\right)+e_{i}\left(t_{j+1}^{i}, j\right)\right)\right)
$$

with the property that $\lim _{i \rightarrow \infty} \varphi_{i}(0,0)=\varphi(0,0),\left\{\varphi_{i}\right\}_{i=0}^{\infty}$ converges graphically to $\varphi^{E}$, for each $i$ we have $\sup _{(t, j) \in \operatorname{dom} e_{i}}\left|e_{i}(t, j)\right|=: \varepsilon_{i}<\infty$, and the sequence $\left\{\varepsilon_{i}\right\}_{i=0}^{\infty}$ converges to 0 .

\subsection{Generalized solutions à la Krasovskii}

To define Krasovskii solutions to $\mathcal{H}$, we define a regularized version of $\mathcal{H}$ "à la Krasovskii". In what follows, $\overline{\mathrm{co}}$ stands for the closed convex hull operation. 
Definition 2.12 (regularized hybrid system $\widehat{\mathcal{H}}$ ). Given a hybrid system $\mathcal{H}=\left(f, g, C, D, \mathbb{R}^{n}\right)$ and a set $O$ satisfying Assumption 2.5, the regularization (à la Krasovskii) of $\mathcal{H}$ is denoted by $\widehat{\mathcal{H}}=(\widehat{f}, \widehat{g}, \widehat{C}, \widehat{D}, O)$ where $\widehat{C}=\bar{C} \cap O, \widehat{D}=\bar{D} \cap O$ while $\widehat{f}: \widehat{C} \rightrightarrows \mathbb{R}^{n}, \widehat{g}: \widehat{D} \rightrightarrows \mathbb{R}^{n}$ are defined by

$$
\forall x \in \widehat{C} \quad \widehat{f}(x):=\bigcap_{\delta>0} \overline{\operatorname{co}} f((x+\delta \mathbb{B}) \cap C), \quad \forall x \in \widehat{D} \quad \widehat{g}(x):=\bigcap_{\delta>0} \overline{g((x+\delta \mathbb{B}) \cap D)} .
$$

Regarding the function $f$, the regularization corresponds to the one proposed by Krasovskii in [31] for discontinuous differential equations. (An equivalent description of $\widehat{f}(x)$ would say that it is the smallest closed convex set containing all limits of $f\left(x_{i}\right)$ as $x_{i} \rightarrow x, x_{i} \in C$. Alternatively, one can first consider a mapping whose graph is the closure of the graph of $f$ relative to $\widehat{C} \times \mathbb{R}^{n}$, and then convexify the values of that mapping.) We note that the regularization of $f$ as proposed by Filippov in [21] ignores the behavior of $f$ on sets of measure zero, and thus proves to be unsuitable for hybrid systems (and even for constrained differential equations). Indeed, for example, a set $C$ with zero measure leads to an "empty" regularization. For further details on the relationship of $\widehat{f}$ to $f$, and the corresponding differential inclusion and equation, see [21,25,26,30]. Regarding $g$, the regularization is the one used in [29]; due to the nature of discrete time, the convexification is not needed. We point out that the regularization need not change the data. For example $\widehat{f}(x)=f(x)$ at each $x \in C$ where $f$ is continuous. On the other hand, $\widehat{f}(x)$ is not a singleton whenever $x$ is a point of discontinuity of $f$. Similar comments apply to $g$ if it is a function. Thus, if both $f$ and $g$ are defined on $O$ and continuous, only the sets $C$ and $D$ are affected by the Krasovskii regularization (and $\hat{f}(x)=f(x)$ for all $x \in \hat{C}, \hat{g}(x)=g(x)$ for all $x \in \hat{D}$ ).

When $\mathcal{H}=(f, g, C, D, O)$ corresponds to a system (2) in the fashion described in Section 2.1 and $O=\mathbb{R}^{m+1}$, the regularization of $\mathcal{H}$ corresponds to regularizing $f_{q}, g_{q}$ and closing $C_{q}, D_{q}$ in $\mathbb{R}^{m}$ for each $q \in Q$. When $\mathcal{H}$ corresponds to a system given by domains, edges, guards, and resets, as described in Section 2.1, the regularization of $\mathcal{H}$ corresponds to closing the values of the domain and guard mappings, and regularizing the resets à la Krasovskii. Similar statements, with relative closures considered, can be made for the general $O$.

Example 2.13. Consider the system from Example 2.1. Most of the data of $\mathcal{H}$ is already "regular" with $O=\mathbb{R}^{3}$. The regularized flow and jump sets are equal to the unregularized ones, i.e. $\widehat{C}=C$ and $\widehat{D}=D$, since those are already closed sets in $O$ (the jump set $D_{1}$ is closed, even though it is a union of three guard sets, two of which are not closed). Since $f_{q}$ is continuous, $\hat{f} \equiv f$. The regularization only alters the jump map $g_{1}$ in the definition of $g$ in (3). Indeed, we get

$$
\widehat{g}_{1}(\xi)=\left\{\begin{array}{ccc}
\left(\begin{array}{l}
\xi \\
2
\end{array}\right) & \text { if } \quad \xi_{1}=0, \xi_{2}<0 \\
\left(\begin{array}{l}
\xi \\
3
\end{array}\right) & \text { if } & \xi_{1}<0, \xi_{2}=0 \\
\left(\begin{array}{c}
\xi \\
\{2,3,4\}
\end{array}\right) & \text { if } & \xi_{1}=0, \xi_{2}=0 .
\end{array}\right.
$$

In particular, $\widehat{g}$ is set valued when $\xi=0, q=1$. Hence, when a solution reaches this point, a jump occurs and $q$ can be mapped to either 2,3 , or 4 .

Following the compact form for hybrid systems $\mathcal{H}=(f, g, C, D, O)$ given in (1), we can write its regularized version $\widehat{\mathcal{H}}=(\widehat{f}, \widehat{g}, \widehat{C}, \widehat{D}, O)$ as

$$
\widehat{\mathcal{H}}:\left\{\begin{array}{cccc}
\dot{x} & \in \widehat{f}(x) & x \in \widehat{C} \\
x^{+} & \in \widehat{g}(x) & x \in \widehat{D} .
\end{array}\right.
$$


Note that the differential equation in $\mathcal{H}$ is replaced by a differential inclusion, since $\widehat{f}: \widehat{C} \rightrightarrows \mathbb{R}^{n}$, by its very definition, is in general a set-valued mapping and not a function. Similarly, even if $g$ is single-valued, $\widehat{g}: \widehat{D} \rightrightarrows \mathbb{R}^{n}$ may turn out set valued. A formal definition of Krasovskii solutions follows.

Definition 2.14 (hybrid Krasovskii solution to $\mathcal{H}$ ). A hybrid $\operatorname{arc} \psi: \operatorname{dom} \psi \rightarrow O$ is a hybrid Krasovskii solution to the hybrid system $\mathcal{H}=(f, g, C, D, O)$ with regularization given by $\widehat{\mathcal{H}}=(\widehat{f}, \widehat{g}, \widehat{C}, \widehat{D}, O)$ if $\psi(0,0) \in \widehat{C} \cup \widehat{D}$ and:

(K1) for all $j \in \mathbb{N}$ and almost all $t$ such that $(t, j) \in \operatorname{dom} \psi$,

$$
\psi(t, j) \in \widehat{C}, \quad \dot{\psi}(t, j) \in \widehat{f}(\psi(t, j)) ;
$$

(K2) for all $(t, j) \in \operatorname{dom} \psi$ such that $(t, j+1) \in \operatorname{dom} \psi$,

$$
\psi(t, j) \in \widehat{D}, \quad \psi(t, j+1) \in \widehat{g}(\psi(t, j)) .
$$

Note that in (K1), if the set of all $t$ such that $(t, j) \in \operatorname{dom} \psi$ is nontrivial (i.e., it has interior), then requiring that $\psi(t, j) \in \widehat{C}$ for almost all $t$ such that $(t, j) \in \operatorname{dom} \psi$ is the same as requiring that this holds for all such $t$ 's. This follows from continuity of $\psi(\cdot, j)$ and $\widehat{C}$ being relatively closed. If there is only one $t$ such that $(t, j) \in \operatorname{dom} \psi$, then (K1) is automatically (and vacuously) satisfied.

Example 2.15. Consider the system $\mathcal{H}$ from Example 2.4. The regularized hybrid system $\widehat{\mathcal{H}}$ with $O=\mathbb{R}^{2}$ has data given by $\widehat{C}=\mathbb{R}^{2}, \widehat{D}=[0,1] \times\{0\}$, while $\widehat{f} \equiv f, \widehat{g} \equiv g$. In particular, there exists an instantaneous Zeno Krasovskii solution given by $x(0, j)=\left(\begin{array}{l}0 \\ 0\end{array}\right)$ for all $j \in \mathbb{N}$. As we noted in Example 2.9, this is also a Hermes solution to $\mathcal{H}$, but is not an execution of $\mathcal{H}$. Another way that Krasovskii solutions differ from executions is that there are Krasovskii solutions from initial points $x^{0}$ with $\left|x^{0}\right|=1$ that flow and then jump $\left(\right.$ as $\left.\left(\begin{array}{l}1 \\ 0\end{array}\right) \in \widehat{D}\right)$ while the unique execution from $x^{0}$ only flows.

As we alluded to in the introduction, stabilization of some nonlinear control systems is not possible with a pure state feedback if solutions are understood à la Krasovskii. In other words, for systems like a nonholonomic integrator or like (8), robust stabilization with pure state feedback is not possible. In contrast, hybrid feedback can achieve robust stabilization for such systems. Let us illustrate this point now; for more details see [38]. The example will also show that Krasovskii regularization can introduce nonuniqueness of solutions, but the new solutions need not be "bad".

Example 2.16. Consider the control system

$$
\dot{\xi}=\left(\begin{array}{c}
-\xi_{2} \\
\xi_{1}
\end{array}\right) u \quad \xi \in C_{\circ}:=\left\{\xi \in \mathbb{R}^{2}|| \xi \mid=1\right\}
$$

where $u \in \mathbb{R}$. We want to render the point $\xi^{*}=\left(\begin{array}{c}0 \\ -1\end{array}\right)$ robustly asymptotically stable on $C_{\circ}$. This is not possible with a classical state feedback, but possible with a hybrid controller. Let $q \in Q:=\{-1,1\}$ be the logic state which is toggled when $\left|\xi_{2}\right| \leq-q \xi_{1}$. The control input $u$ is given by the feedback $\kappa(\xi, q)$ which, for each $q \in Q$, is a continuous function of $\xi$ and steers the trajectories to $\xi^{*}$ as shown in Figure 3. The closed-loop system is

$$
f(x)=\left(\begin{array}{c}
-\xi_{2} \\
\xi_{1}
\end{array}\right) \kappa(\xi, q), g(x)=\left(\begin{array}{c}
\xi \\
-q
\end{array}\right), C=\left\{(\xi, q) \in \mathbb{R}^{2} \times Q\left|\xi \in C_{\circ},\right| \xi_{2} \mid \geq-q \xi_{1}\right\}, D=\left(C_{\circ} \times Q\right) \backslash C .
$$

Denote this system $\mathcal{H}$. Note that $C \cup D=C_{\circ} \times Q$ and that $C \cap D=\emptyset$. The Krasovskii regularization of $\mathcal{H}$ with $O=\mathbb{R}^{3}$ is given by $\widehat{\mathcal{H}}=\left(\widehat{f}, \widehat{g}, \widehat{C}, \widehat{D}, \mathbb{R}^{3}\right)$ where $\widehat{f} \equiv f, \widehat{g} \equiv g, \widehat{C}=C$, and $\widehat{D}=\bar{D}$. Krasovskii solutions 


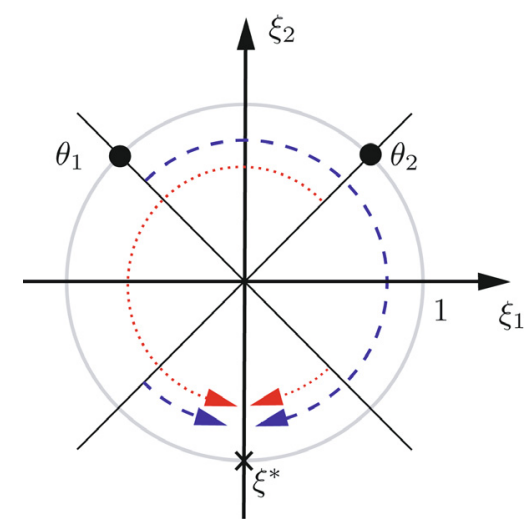

Figure 3. Global stabilization of a point in a circle. Solutions to the hybrid system $\mathcal{H}$ in Example 2.16 are steered to $\xi^{*}$ in the direction indicated with the arrows for $q=1$ (dashed) and for $q=-1$ (dotted).

(and executions) coincide with CADLAG solutions to $\mathcal{H}$ from every point in $C$ except from $\left\{\theta_{1}\right\} \times\{1\}$ and $\left\{\theta_{2}\right\} \times\{-1\}$. From these points, CADLAG solutions and executions are unique, while Krasovskii solutions are not: one can flow and correspond to a CADLAG solution, another can immediately jump. Both eventually flow towards $\xi^{*}$. (One cannot really talk about CADLAG solutions from $D$; in other words, they need to be initialized with a correct logic state.) Such nonuniqueness can arise for CADLAG solutions when (arbitrarily small) measurement noise is present. Note though that $\xi^{*}$ is asymptotically stable when Krasovskii solutions are considered. In a sense, this also means that $\xi^{*}$ is robustly asymptotically stable when CADLAG solutions are considered.

With the stated mild assumptions on $f$ and $g$, the system $\widehat{\mathcal{H}}=(\widehat{f}, \widehat{g}, \widehat{C}, \widehat{D}, O)$ has the regularity properties (stated below, in Th. 2.17) that were imposed on the hybrid systems by the authors et al. in [23,24] and led to results on sequential compactness of the sets of solutions to hybrid systems. In particular, such properties guarantee that an appropriately understood limit of a sequence of solutions to a hybrid system is itself a solution.

Below, we say that a set-valued mapping $\phi: S \rightrightarrows \mathbb{R}^{n}$, where $S \subset O$, is outer semicontinuous relative to $S$ if for any $x \in S$ and any sequence $\left\{x_{i}\right\}_{i=1}^{\infty}$ with $x_{i} \in S, \lim _{i \rightarrow \infty} x_{i}=x$ and any sequence $\left\{y_{i}\right\}_{i=1}^{\infty}$ with $y_{i} \in \phi\left(x_{i}\right)$ and $\lim _{i \rightarrow \infty} y_{i}=y$ we have $y \in \phi(x)$.

Theorem 2.17 (basic properties of $\widehat{\mathcal{H}})$. Under Assumption 2.5 , the regularized hybrid system $\widehat{\mathcal{H}}=(\widehat{f}, \widehat{g}, \widehat{C}, \widehat{D}, O)$ satisfies:

(A0) $O \subset \mathbb{R}^{n}$ is an open set.

(A1) $\widehat{C}$ and $\widehat{D}$ are relatively closed sets in $O$.

(A2) $\widehat{f}: \widehat{C} \rightrightarrows \mathbb{R}^{n}$ is outer semicontinuous relative to $\widehat{C}$ and locally bounded on $O$, and for all $x \in \widehat{C}$, $\widehat{f}(x)$ is nonempty and convex.

(A3) $\widehat{g}: \widehat{D} \rightrightarrows \mathbb{R}^{n}$ is outer semicontinuous relative to $\widehat{D}$ and locally bounded on $O$, and for all $x \in \widehat{D}$, $\widehat{g}(x)$ is nonempty and $\widehat{g}(x) \subset O$.

One could say that $\widehat{f}, \widehat{g}$ are "minimal" among all set-valued mappings $\tilde{f}, \tilde{g}$ possessing the properties in (A2), (A3) and such that $f(x) \in \tilde{f}(x)$ for all $x \in C, g(x) \subset \tilde{g}(x)$ for all $x \in D$. Similarly, $\widehat{C}, \widehat{D}$ are the smallest relatively closed subsets of $O$ containing $C \cap O, D \cap O$, respectively ${ }^{4}$ In particular, if $f$ and $g$ have

\footnotetext{
4 The basic properties of $\widehat{\mathcal{H}}$ also illustrate some advantages of considering an open state space $O \neq \mathbb{R}^{n}$. When one wants to restrict a hybrid system to an open and invariant subset of the original state space $\mathbb{R}^{n}$, say the basin of attraction of a compact and locally asymptotically stable set, it is impossible to do with the modeling capabilities of $\widehat{C}$ and $\widehat{D}$ while at the same time preserving their closeness (or relative closeness).
} 
the properties (A2) and (A3) (note that these properties make sense for mappings defined on $C$ and $D$, and not on subsets of $O$ ) while $C \cap O$ and $D \cap O$ are relatively closed in $O$, then $f(x)=\widehat{f}(x)$ for $x \in \widehat{C}, g(x)=\widehat{g}(x)$ for $x \in \widehat{D}$ while $\widehat{C}=C \cap O, \widehat{D}=D \cap O$. In such a case, Krasovskii solutions to $\mathcal{H}=(f, g, C, D, O)$ are exactly the solutions to $\mathcal{H}=(f, g, C, D, O)$ considered in [23]. Thus, it was the Krasovskii concept of a generalized solution that made possible the robust stability theory developments in $[12,23,41]$.

One of the benefits of the properties in Theorem 2.17 is that, for systems that possess them, very general conditions for existence of solutions can be given, and maximal solutions behave as expected: that is, they are either complete or "blow up" in finite hybrid time (a solution is complete if its domain is unbounded). More specifically, under Assumption 2.5, and hence in the presence of the properties listed in Theorem 2.17, the following is true. If $\psi^{0} \in \widehat{D}$ or the following condition holds:

(VC) $\psi^{0} \in \widehat{C}$ and for some neighborhood $U$ of $\psi^{0}$, for all $\psi^{\prime} \in U \cap \widehat{C}, T_{\widehat{C}}\left(\psi^{\prime}\right) \cap \widehat{f}\left(\psi^{\prime}\right) \neq \emptyset$,

where $T_{\widehat{C}}\left(\psi^{\prime}\right)$ is the tangent cone to $\widehat{C}$ at $\psi^{\prime}$, then there exists a nontrivial Krasovskii solution $\psi$ to $\mathcal{H}$ with $\psi(0,0)=\psi^{0}$. If (VC) holds for all $\psi^{0} \in \widehat{C} \backslash \widehat{D}$, then for any maximal solution $\psi$ with $\psi(0,0)=\psi^{0}$ (a Krasovskii solution $\psi$ is said to be maximal if there does not exist another Krasovskii solution $\psi^{\prime}$ such that $\psi$ is a truncation of $\psi^{\prime}$ to some proper subset of $\operatorname{dom} \psi^{\prime}$ ) at least one of the following statements is true:

(i) $\psi$ is complete;

(ii) $\psi$ eventually leaves every compact subset of $O$ : for any compact $K \subset O$, there exists $(T, J) \in \operatorname{dom} \psi$ such that for all $(t, j) \in \operatorname{dom} \psi$ with $(T, J) \prec(t, j), \psi(t, j) \notin K$;

(iii) for some $(T, J) \in \operatorname{dom} \psi,(T, J) \neq(0,0)$, we have $\psi(T, J) \notin \widehat{C} \cup \widehat{D}$.

If additionally

(VD) for all $\psi^{0} \in \widehat{D}, \widehat{g}\left(\psi^{0}\right) \subset \widehat{C} \cup \widehat{D}$,

then case (iii) above does not occur. For details, see [23], Proposition 2.5, or the viability conditions in [3].

Note that the viability condition (VC) for the continuous evolution is automatically satisfied at each point $\psi^{0}$ in the interior of $\widehat{C}$. Therefore, when $\widehat{C} \cup \widehat{D}=O$ (a condition that is common in many models for hybrid systems, see the Introduction), (VC) holds for all $\psi^{0} \in \widehat{C} \backslash \widehat{D}$ since $\widehat{C} \backslash \widehat{D}=O \backslash \widehat{D}$ and the latter set is open. Consequently, if $\widehat{C} \cup \widehat{D}=O$, for all $\psi^{0} \in O$ there exists a nontrivial solution $\psi$ with $\psi(0,0)=\psi^{0}$, and for any maximal solution, either (i) or (ii) above holds.

We add that, if the properties in Theorem 2.17 are present for a hybrid system, then Krasovskii solutions to that system agree with executions; recall the definition above Example 2.4. Furthermore, it is then irrelevant whether one requests, in the definition of an execution, that $\tilde{\xi}(t, j) \in C$ for all $t \in\left[t_{j}, t_{j+1}\right)$ or for all $t \in\left[t_{j}, t_{j+1}\right]$, as used, for example, in [34] and [3], respectively. (This comment applies whenever the flow set is relatively closed in the state space.) For implications of the closeness assumptions on the properties of solution sets like computability, we point the reader to [18].

To conclude this section, let us give two examples that will show that Krasovskii solutions may introduce undesired solutions (and thus, in light of the results of the next section, can detect nonrobustness of control algorithms) and that with appropriate modeling, sometimes the undesired solutions can be eliminated.

Example 2.18. Consider the "rotate and dissipate" example in [24], Section 2, which consists of a planar system with trajectories that rotate continuously (clockwise) until they hit the $\xi_{2}$-axis at which point they rotate instantaneously to the $\xi_{1}$-axis, decreasing in magnitude by the factor $\mu \in(0,1)$. This process is recurrent and, in this way, these trajectories tend to the origin. The flows of the "rotate and dissipate" system are given by

$$
\dot{\xi}=f(\xi):=\left(\begin{array}{c}
\xi_{2} \\
-\xi_{1}
\end{array}\right) \quad \xi \in C:=\left\{\xi \in \mathbb{R}^{2} \mid \xi_{1} \neq 0\right\}
$$

and the jumps are governed by

$$
\xi^{+}=g(\xi):=\left(\begin{array}{c}
\mu \xi_{2} \\
0
\end{array}\right) \quad \xi \in D:=\left\{\xi \in \mathbb{R}^{2} \mid \xi_{1}=0\right\} .
$$




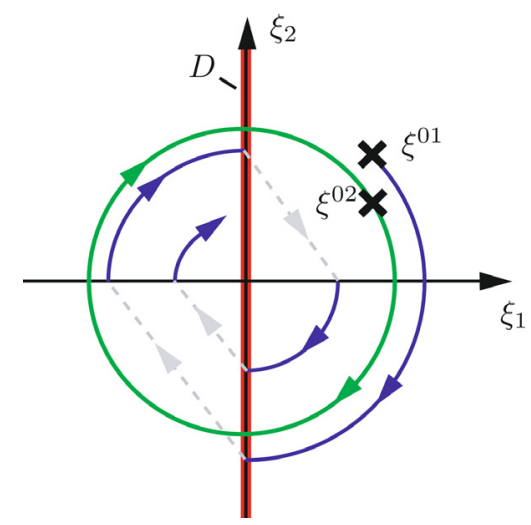

Figure 4. Krasovskii solutions to $\mathcal{H}$ in Example 2.18 fail to converge to the origin. The CADLAG solution starting from $\xi^{01}$ jumps when the $\xi_{2}$-axis is crossed. Krasovskii solutions, like the one starting from $\xi^{02}$, may "miss" the jumps and therefore, never approach the origin.

Hybrid systems with this type of flow and jump sets arise in several control engineering applications, see $e . g$. the reset and impulsive control system model in Example 6.1 and the hybrid control system in Example 6.2. We denote this hybrid system by $\mathcal{H}=\left(f, g, C, D, \mathbb{R}^{2}\right)$. The Krasovskii regularization of $\mathcal{H}$ with $O=\mathbb{R}^{2}$ is given by $\widehat{\mathcal{H}}=\left(\widehat{f}, \widehat{g}, \widehat{C}, \widehat{D}, \mathbb{R}^{2}\right)$ where $\widehat{f} \equiv f, \widehat{g} \equiv g, \widehat{C}=\mathbb{R}^{2}$, and $\widehat{D}=D$. Consequently, there exist Krasovskii solutions to $\mathcal{H}$ that flow for all time and never jump. These additional Krasovskii solutions that do not converge to the origin reflect the effects of (arbitrarily small) measurement noise that can cause CADLAG solutions or executions to "miss" the jump set as show in Figure 4. (Such noise can be constructed similarly to what we outlined in Ex. 2.9.)

Example 2.19. Consider the system of Example 2.18. We will use a zero-cross detection (ZCD) algorithm to enforce the jumps when Krasovskii solutions hit the $\xi_{2}$-axis. Let $q \in Q:=\{-1,1\}$ be a logic state that stores the sign of $\xi_{1}$ (we will let $\operatorname{sign}(r)=-1$ if $r<0,1$ if $r>0$, and $\{-1,1\}$ if $r=0$ ). The ZCD implementation of the "rotate and dissipate" system with state $x:=\left(\begin{array}{l}\xi \\ q\end{array}\right)$ has flows governed by

$$
\dot{x}=f(x):=\left(\begin{array}{c}
\xi_{2} \\
-\xi_{1} \\
0
\end{array}\right) \quad x \in C:=\left\{(\xi, q) \in \mathbb{R}^{2} \times Q \mid \xi_{1} q \geq 0\right\}
$$

and jumps governed by

$$
x^{+} \in g(x):=\left(\begin{array}{c}
\mu \xi_{2} \\
0 \\
\operatorname{sign}\left(\mu \xi_{2}\right)
\end{array}\right) \quad x \in D:=\bigcup_{q \in Q}\left(D_{q} \times\{q\}\right)
$$

where $D_{-1}:=\left\{\xi \in \mathbb{R}^{2} \mid \xi_{1}=0, \xi_{2} \geq 0\right\}$ and $D_{1}:=\left\{\xi \in \mathbb{R}^{2} \mid \xi_{1}=0, \xi_{2} \leq 0\right\}$. We denote this hybrid system by $\mathcal{H}=(f, g, C, D)$. Solutions to $\mathcal{H}$ starting with $q^{0}=\operatorname{sign}\left(\xi_{1}^{0}\right)$ rotate continuously (clockwise) until they hit the $\xi_{2}$-axis. Flow is then no longer possible due to the condition $\xi_{1} q \geq 0$; hence, a jump is enforced. This is depicted in Figure 5. We note that the Krasovskii regularization of $\mathcal{H}$ with $O=\mathbb{R}^{3}$ is equal to $\mathcal{H}$ itself since the data $(f, g, C, D)$ has all the regularity properties in Definition 2.12 . 


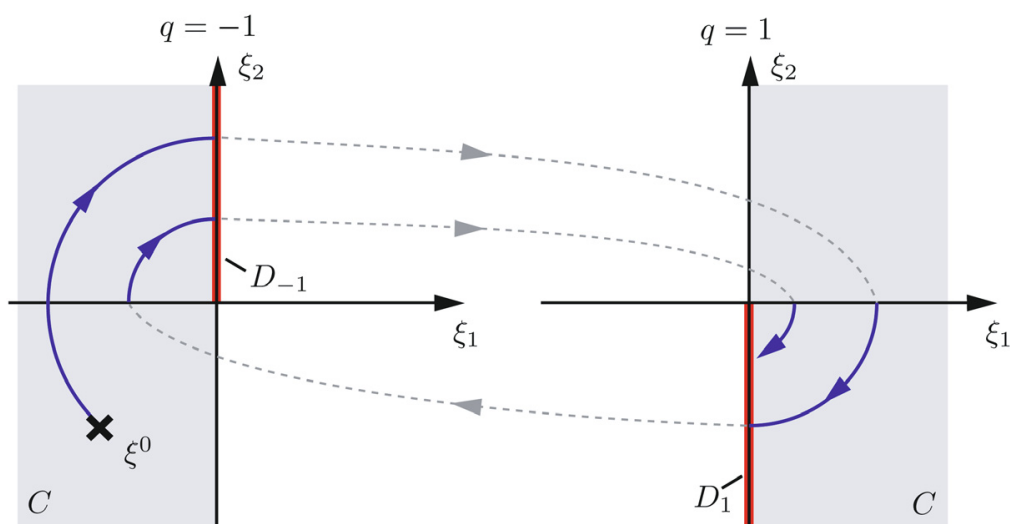

Figure 5. Jumps are enforced when solutions to $\mathcal{H}$ in Example 2.19 tend to cross the $\xi_{2}$-axis. At jumps, the logic state $q$ is toggled by the sign function and flows are enabled in the complementary (closed) right-half plane. A solution starting at $\xi^{0}$ (solid, blue) and the transitions from one mode to the other (dashed) are depicted.

\section{Statement of MAin ReSults}

We now extend the work of Hermes [26] and Hàjek [25] to the hybrid setting, and show that hybrid Krasovskii solutions to $\mathcal{H}$, given in Definition 2.14, are equivalent to hybrid Hermes solutions to $\mathcal{H}$, given in Definition 2.7.

Theorem 3.1 (Krasovskii solutions $\equiv$ Hermes solutions). Under Assumption 2.5, a hybrid arc is a hybrid Krasovskii solution to $\mathcal{H}$ if and only if it is a hybrid Hermes solution to $\mathcal{H}$.

The two implications are stated and proved as Corollary 4.4 and Corollary 5.2 in Section 4.1 and Section 5 , respectively.

We note that Theorem 3.1 generalizes, to the hybrid framework, the result by Hàjek [25] given for differential equations. In proving the theorem, we first extend some results by Hàjek to differential equations with a constraint (and we give a proof quite different from that by Hàjek). We will also rely on results on perturbations of hybrid systems given in [23].

The next result is a generalization to the hybrid framework of the result given by Coron and Rosier [19] in the context of robust stabilizability of nonlinear systems with time-varying feedback laws. Recall that hybrid control-Hermes solutions were given in Definition 2.11.

Theorem 3.2 (Krasovskii solutions $\equiv$ control-Hermes solutions). Under Assumption 2.10, for a hybrid system $\mathcal{H}$ with $f$ and $g$ given by (5), a hybrid arc is a hybrid Krasovskii solution to $\mathcal{H}$ if and only if it is a hybrid control-Hermes solution to $\mathcal{H}$.

The two implications are given as Corollary 4.7 and Proposition 5.1 in Section 4.2 and Section 5 , respectively. (One of them naturally follows from Th. 3.1.)

\section{Krasovskit solutions are Hermes / COntrol-Hermes solutions}

In this section, via several intermediate results, we will show that Krasovskii solutions are Hermes, and for the case when measurement noise appears in some but not every component of the state, control Hermes. This will amount to showing one direction of the equivalences in Theorems 3.1 and 3.2. 


\subsection{Krasovskii solutions are Hermes}

Theorem 4.2 below is an extension of Theorem 5.5 in [25] to differential equations with a constraint $x(t) \in C$. Our proof is also different (and shorter) than that given in [25]. We will need the following corollary of [25], Lemma 3.3.

Lemma 4.1. Let $f$ satisfy Assumption 2.5 and let $\psi:[0, T] \rightarrow O$ be an absolutely continuous function that satisfies, for almost all $t \in[0, T], \dot{\psi}(t) \in \widehat{f}(\psi(t))$. Then, for every $\varepsilon>0$, there is a closed null-set $Z$ and a Lipschitz function $z:[0, T] \rightarrow O$ such that $|\psi(t)-z(t)| \leq \varepsilon$ for all $t \in[0, T]$ and which is piecewise linear in the sense that $\dot{z}(t)=\dot{\psi}(\alpha)$ for all $t$ in any component $(\alpha, \beta)$ of $[0, T] \backslash Z$, and for any such component, $\dot{\psi}(\alpha) \in \widehat{f}(\psi(\alpha))$.

Above, by a component of (the open set) $[0, T] \backslash Z$ we understand any of the (at most countably many) mutually disjoint intervals $(\alpha, \beta)$ covering $[0, T] \backslash Z$. In what follows, we will refer to the family of all such intervals as $\mathcal{I}$. Below, we will also use the term "piecewise constant" to mean that a function is constant on each $(\alpha, \beta) \in \mathcal{I}$. Finally, we note that directly from the proof of the result above one can deduce that the size of the intervals $(\alpha, \beta)$ in $\mathcal{I}$ can be made arbitrarily small.

Theorem 4.2. Let $f$ satisfy Assumption 2.5 and let $\psi:[0, T] \rightarrow O$ be an absolutely continuous function that satisfies, for almost all $t \in[0, T], \psi(t) \in \widehat{C}, \dot{\psi}(t) \in \widehat{f}(\psi(t))$. Then, for any $\varepsilon>0$ such that rge $\psi+\varepsilon \mathbb{B} \subset O$ and any $\varphi_{0} \in C$ such that $\left|\varphi_{0}-\psi(0)\right|<\varepsilon$, there exists a Lipschitz and piecewise linear $\varphi:[0, T] \rightarrow O$ with $\varphi(0)=\varphi_{0}$ and a measurable $e:[0, T] \rightarrow \mathbb{R}^{n}$ such that $\sup _{t \in[0, T]}|e(t)| \leq \varepsilon, \varphi+e$ is piecewise constant on $[0, T]$,

$$
\varphi(t)+e(t) \in C \quad \text { for all } t \in[0, T], \quad \dot{\varphi}(t)=f(\varphi(t)+e(t)) \text { for almost all } t \in[0, T]
$$

and $|\psi(t)-\varphi(t)| \leq \varepsilon$ for all $t \in[0, T]$.

Proof. Fix $\varepsilon>0$ so that rge $\psi+\varepsilon \mathbb{B} \subset O$, pick $\varphi_{0} \in C$ so that $\left|\varphi_{0}-\psi(0)\right|<\varepsilon$, and pick any $\varepsilon^{\prime} \in\left(0, \varepsilon-\left|\varphi_{0}-\psi(0)\right|\right)$. Let $L>0$ be a bound on $f$ on $\operatorname{rge} \psi+\varepsilon^{\prime} \mathbb{B}$ (so in particular, $L$ is a Lipschitz constant for $\psi$ ). Use Lemma 4.1 to obtain $z:[0, T] \rightarrow O$ with $|\psi(t)-z(t)| \leq \varepsilon^{\prime} / 8$ in a way that $\beta-\alpha \leq \frac{\varepsilon^{\prime}}{8(1+L)}$ for each component $(\alpha, \beta)$ of $[0, T] \backslash Z$. Then, in particular, $|\psi(t)-\psi(\alpha)| \leq \varepsilon^{\prime} / 8$ for all $t \in(\alpha, \beta)$ and all intervals $(\alpha, \beta)$ in $\mathcal{I}$. For each segment $(\alpha, \beta) \in \mathcal{I}$, by the definition of $\widehat{f}$ and since $\dot{\psi}(\alpha) \in \widehat{f}(\psi(\alpha))$, there exist points $\psi_{j}^{\alpha} \in C, j=1,2, \ldots, n+1$, so that $\left|\psi_{j}^{\alpha}-\psi(\alpha)\right| \leq \varepsilon^{\prime} / 8$ and constants $\lambda_{j}^{\alpha} \geq 0, j=1,2, \ldots, n+1$, so that $\sum_{j=1}^{n+1} \lambda_{j}^{\alpha}=1$ and

$$
\left|\left(\sum_{j=1}^{n+1} \lambda_{j}^{\alpha} f\left(\psi_{j}^{\alpha}\right)\right)-\dot{\psi}(\alpha)\right| \leq \frac{\varepsilon^{\prime}}{8(1+T)} .
$$

Divide $(\alpha, \beta)$ into $n+1$ subintervals $\left(\gamma_{j-1}^{\alpha}, \gamma_{j}^{\alpha}\right)$ with $\gamma_{0}^{\alpha}=\alpha, \gamma_{n+1}^{\alpha}=\beta$ of lengths $\lambda_{j}^{\alpha}(\beta-\alpha)$. Now define a piecewise linear function $v:[0, T] \rightarrow \mathbb{R}^{n}$ almost everywhere by $v(t)=f\left(\psi_{j}^{\alpha}\right)$ on $\left(\gamma_{j-1}^{\alpha}, \gamma_{j}^{\alpha}\right)$. Note that $v \in L_{1}[0, T]$, and consequently, the function $\varphi:[0, T] \rightarrow \mathbb{R}^{n}$ defined by

$$
\varphi(t)=\varphi_{0}+\int_{0}^{t} v(\tau) \mathrm{d} \tau
$$

is absolutely continuous. Furthermore, $\dot{\varphi}(t)=v(t)$ for almost all $t \in[0, T]$ (in fact, whenever $t \in\left(\gamma_{j-1}^{\alpha}, \gamma_{j}^{\alpha}\right)$, $j=1,2, \ldots, n+1)$. Also, define $e:[0, T] \rightarrow \mathbb{R}^{n}$ as follows: set

$$
e(t)=\psi_{j}^{\alpha}-\varphi(t) \quad \text { if } \quad t \in\left(\gamma_{j-1}^{\alpha}, \gamma_{j}^{\alpha}\right)
$$

and note that this defines $e$ for almost all $t \in[0, T]$, and that for those $t$ 's, we have $\dot{\varphi}(t)=f(\varphi(t)+e(t))$ as well as $\varphi(t)+e(t) \in C$. For each $t$ at which $e(t)$ has not been defined yet, one can find $c_{t} \in C$ such 
that $\left|c_{t}-\psi(t)\right|<\varepsilon^{\prime} / 4$ and then set $e(t)=c-\varphi(t)$. Then $\varphi(t)+e(t) \in C$ for all $t \in[0, T]$. Also, $\varphi(t)+e(t)$ is constant on each $\left(\gamma_{j-1}^{\alpha}, \gamma_{j}^{\alpha}\right)$, as so $\varphi+e$ is piecewise constant on $[0, T]$. We claim that $\varphi$ and $e$ have the other desired properties.

For each initial point $\bar{\alpha}$ of some interval $(\alpha, \beta) \in \mathcal{I}$

$$
\begin{aligned}
|\varphi(\bar{\alpha})-z(\bar{\alpha})| & \leq|\varphi(0)-z(0)|+\left|\int_{0}^{\bar{\alpha}}(\dot{\varphi}(t)-\dot{z}(t)) \mathrm{d} t\right| \\
& \leq\left|\varphi_{0}-z(0)\right|+\sum_{(\alpha, \beta) \in \mathcal{I}, \beta \leq \bar{\alpha}}\left|\int_{\alpha}^{\beta} \dot{\varphi}(t) \mathrm{d} t-\dot{\psi}(\alpha)(\beta-\alpha)\right| \\
& \leq\left|\varphi_{0}-\psi(0)\right|+|\psi(0)-z(0)|+\sum_{(\alpha, \beta) \in \mathcal{I}, \beta \leq \bar{\alpha}} \frac{\varepsilon^{\prime}(\beta-\alpha)}{8(1+T)} \\
& \leq\left|\varphi_{0}-\psi(0)\right|+\varepsilon^{\prime} / 8+\frac{\varepsilon^{\prime}}{8(1+T)} \sum_{(\alpha, \beta) \in \mathcal{I}, \beta \leq \bar{\alpha}}(\beta-\alpha) \\
& =\left|\varphi_{0}-\psi(0)\right|+\varepsilon^{\prime} / 8+\frac{\varepsilon^{\prime}}{8(1+T)} \bar{\alpha} \\
& \leq\left|\varphi_{0}-\psi(0)\right|+\varepsilon^{\prime} / 4
\end{aligned}
$$

where the sum above is over all intervals $(\alpha, \beta) \in \mathcal{I}$ with $\beta \leq \bar{\alpha}$ (so that in particular, $\sum(\beta-\alpha)=\bar{\alpha}$ ) and the last inequality above relies on $\bar{\alpha}<T^{5}$. Furthermore, for each interval $(\alpha, \beta)$ and any $\tau \in(\alpha, \beta)$,

$$
\left|\int_{\alpha}^{\tau} \dot{\varphi}(t) \mathrm{d} t-\dot{\psi}(\alpha)(\tau-\alpha)\right| \leq 2 L(\tau-\alpha) \leq 2 L(\beta-\alpha) \leq \varepsilon^{\prime} / 4
$$

since both $\dot{\varphi}$ and $\dot{\psi}$ are bounded by $L$. Consequently,

$$
|\varphi(t)-z(t)| \leq\left|\varphi_{0}-\psi(0)\right|+\varepsilon^{\prime} / 2
$$

for all $t \in[0, T]$, and hence

for all $t \in[0, T]$.

$$
|\varphi(t)-\psi(t)| \leq\left|\varphi_{0}-\psi(0)\right|+5 \varepsilon^{\prime} / 8<\varepsilon
$$

For the error bound, one obtains, for $t$ in one of the intervals $\left(\gamma_{j-1}^{\alpha}, \gamma_{j}^{\alpha}\right)$,

$$
\begin{aligned}
|e(t)| & \leq\left|\psi_{j}^{\alpha}-\psi(\alpha)\right|+|\psi(\alpha)-\psi(t)|+|\psi(t)-\varphi(t)| \\
& \leq \varepsilon^{\prime} / 8+\varepsilon^{\prime} / 8+\left|\varphi_{0}-\psi(0)\right|+5 \varepsilon^{\prime} / 8 \\
& =\left|\varphi_{0}-\psi(0)\right|+7 \varepsilon^{\prime} / 8<\varepsilon .
\end{aligned}
$$

For the remaining $t$ 's,

$$
|e(t)| \leq\left|c_{t}-\psi(t)\right|+|\psi(t)-\varphi(t)| \leq \varepsilon^{\prime} / 4+\left|\varphi_{0}-\psi(0)\right|+5 \varepsilon^{\prime} / 8<\varepsilon
$$

by the choice of $c_{t}$ and the previously established bound on $|\psi(t)-\varphi(t)|$. Thus $|e(t)| \leq \varepsilon$ for all $t \in[0, T]$.

${ }^{5}$ We used the fact that by the construction of $v$, for any $(\alpha, \beta)$ in $\mathcal{I}$,

and thus

$$
\int_{\alpha}^{\beta} \dot{\varphi}(t) \mathrm{d} t=\sum_{j=1}^{n+1} \int_{\gamma_{j-1}^{\alpha}}^{\gamma_{j}^{\alpha}} v(t) \mathrm{d} t=\sum_{j=1}^{n+1} \lambda_{j}^{\alpha}(\beta-\alpha) f\left(\psi_{j}^{\alpha}\right)=(\beta-\alpha) \sum_{j=1}^{n+1} \lambda_{j}^{\alpha} f\left(\psi_{j}^{\alpha}\right)
$$

$$
\left|\int_{\alpha}^{\beta} \dot{\varphi}(t) \mathrm{d} t-\dot{\psi}(\alpha)(\beta-\alpha)\right| \leq(\beta-\alpha)\left|\sum_{j=1}^{n+1} \lambda_{j}^{\alpha} f\left(\psi_{j}^{\alpha}\right)-\dot{\psi}(\alpha)\right| \leq \frac{\varepsilon^{\prime}(\beta-\alpha)}{8(1+T)}
$$


Theorem 4.3. Let Assumption 2.5 hold and let $\psi: \operatorname{dom} \psi \rightarrow O$ be a hybrid Krasovskii solution to $\mathcal{H}=$ $(f, g, C, D, O)$. Pick any $\varepsilon>0$ and any $(T, J) \in \operatorname{dom} \psi$. Let $E=\operatorname{dom} \psi \cap([0, T] \times\{0,1, \ldots, J\})$ and express $E$ as $\bigcup_{j=0}^{J}\left(\left[t_{j}, t_{j+1}\right] \times\{j\}\right)$. Then, there exist a measurable function $e: \operatorname{dom} e \rightarrow \mathbb{R}^{n}$, dom $e=E$ and a hybrid $\operatorname{arc} \varphi: \operatorname{dom} \varphi \rightarrow O, \varphi(0,0)=\psi(0,0), \operatorname{dom} \varphi=E$ such that $\sup _{(t, j) \in \operatorname{dom} e}|e(t, j)| \leq \varepsilon$ and

(V1) for all $j=0,1, \ldots, J$,

$$
\varphi(t, j)+e(t, j) \in C \text { for all } t \in\left[t_{j}, t_{j+1}\right), \quad \dot{\varphi}(t, j)=f(\varphi(t, j)+e(t, j)) \text { for almost all } t \in\left[t_{j}, t_{j+1}\right] ;
$$

(V2) for all $j=0,1, \ldots, J-1$,

$$
\varphi\left(t_{j+1}, j\right)+e\left(t_{j+1}, j\right) \in D, \quad \varphi\left(t_{j+1}, j+1\right) \in g\left(\varphi\left(t_{j+1}, j\right)+e(t, j)\right)
$$

and $|\varphi(t, j)-\psi(t, j)| \leq \varepsilon$ for every $(t, j) \in \operatorname{dom} \varphi$.

Proof. Fix $\varepsilon>0$ small enough so that $\psi(t, j)+\varepsilon \mathbb{B} \subset O$ for all $(t, j) \in E$. Note that for each $j=0,1, \ldots, J-1$ there exists $z_{j} \in D$ and $w_{j+1} \in g\left(z_{j}\right)$ such that $\left|z_{j}-\psi\left(t_{j+1}, j\right)\right| \leq \frac{\varepsilon}{8}$ and $\left|w_{j+1}-\psi\left(t_{j+1}, j+1\right)\right| \leq \frac{\varepsilon}{8}$. Indeed, for any $x \in \widehat{D}$ and $\epsilon>0$, we have:

$$
\widehat{g}(x) \subset \overline{g((x+\epsilon \mathbb{B}) \cap D)} \cap O \subset(g((x+\epsilon \mathbb{B}) \cap D)+\epsilon \mathbb{B}) \cap O,
$$

where the first inclusion above comes from the definition of $\widehat{g}(x)$, and the second one from the definition of the closure of a set. Consequently, for each $y \in \widehat{g}(x)$, there exists $z \in(x+\epsilon \mathbb{B}) \cap D$ and $w \in g(z)$ such that $y \in g(y)+\epsilon \mathbb{B}$.

On each segment $\left[t_{j}, t_{j+1}\right] \times\{j\}$ that is nontrivial, i.e., with $t_{j+1}>t_{j}$, by Theorem 4.2 , there exists an absolutely continuous function $\varphi_{j}:\left[t_{j}, t_{j+1}\right] \rightarrow O$ starting at $\varphi_{j}^{0} \in \psi\left(t_{j}, j\right)+\frac{\varepsilon}{8} \mathbb{B}$, where $\varphi_{j}^{0}=w_{j}$ if $j>0$, $\varphi_{j}^{0}=\psi(0,0)$ if $j=0$, and a measurable function $e_{j}:\left[t_{j}, t_{j+1}\right] \rightarrow \mathbb{R}^{n}$ with $\sup _{t \in\left[t_{j}, t_{j+1}\right]}\left|e_{j}(t)\right| \leq \frac{\varepsilon}{4}$ satisfying

$$
\varphi_{j}(t)+e_{j}(t) \in C \text { for all } t \in\left[t_{j}, t_{j+1}\right], \quad \dot{\varphi}_{j}(t)=f\left(\varphi_{j}(t)+e_{j}(t)\right) \text { for almost all } t \in\left[t_{j}, t_{j+1}\right]
$$

and such that

$$
\left|\varphi_{j}(t)-\psi(t, j)\right| \leq \frac{\varepsilon}{4} \quad \text { for all } t \in\left[t_{j}, t_{j+1}\right]
$$

We now construct the measurement noise $e$. On each segment $\left[t_{j}, t_{j+1}\right] \times\{j\} \subset E$ that is trivial, i.e. segments with $t_{j+1}=t_{j}$, let $e_{j}^{\prime}=z_{j}-w_{j}$ if $J>j>0, e_{j}^{\prime}=z_{0}-\psi(0,0)$ if $j=0$, and $e_{j}^{\prime}=0$ if $j=J$. On each nontrivial segment $\left[t_{j}, t_{j+1}\right] \times\{j\}$, let $e_{j}^{\prime}(t)=e_{j}(t)$ for all $(t, j) \in\left[t_{j}, t_{j+1}\right) \times\{j\}, e_{j}^{\prime}\left(t_{j+1}\right)=z_{j}-\varphi_{j}\left(t_{j+1}\right)$ if $j<J$, $e_{j}^{\prime}\left(t_{j+1}\right)=0$ if $j=J$.

Then, $\varphi$ and $e$ are given by

$$
\begin{aligned}
\varphi(t, j) & := \begin{cases}\varphi_{j}(t) & \text { if } \quad(t, j) \in\left[t_{j}, t_{j+1}\right] \times\{j\} \text { nontrivial } \\
w_{j} & \text { if } \quad(t, j)=\left[t_{j}, t_{j+1}\right] \times\{j\} \text { trivial }\end{cases} \\
e(t, j) & := \begin{cases}e_{j}^{\prime}(t) & \text { if } \quad(t, j) \in\left[t_{j}, t_{j+1}\right] \times\{j\} \text { nontrivial } \\
e_{j}^{\prime} & \text { if } \quad(t, j)=\left[t_{j}, t_{j+1}\right] \times\{j\} \text { trivial. }\end{cases}
\end{aligned}
$$

By construction, $\varphi$ and $e$ satisfy (V1) and (V2). It follows that $|\varphi(t, j)-\psi(t, j)| \leq \varepsilon$ for all $(t, j) \in \operatorname{dom} \varphi$ since on every trivial segment

$$
|\varphi(t, j)-\psi(t, j)|=\left|w_{j}-\psi(t, j)\right| \leq \frac{\varepsilon}{8}
$$

and on every nontrivial segment $\left[t_{j}, t_{j+1}\right] \times\{j\}$

$$
|\varphi(t, j)-\psi(t, j)|=\left|\varphi_{j}(t)-\psi(t, j)\right| \leq \frac{\varepsilon}{4} \quad \forall t \in\left[t_{j}, t_{j+1}\right] .
$$


Moreover, $\sup _{(t, j) \in \operatorname{dom} e}|e(t, j)| \leq \varepsilon$ since on every trivial interval

$$
\begin{aligned}
|e(t, j)| & =\left|z_{j}-w_{j}\right| \leq\left|z_{j}-\psi(t, j)\right|+\left|\psi(t, j)-w_{j}\right| \leq \frac{\varepsilon}{4} & & \text { if } J>j>0 \\
|e(t, j)| & =\left|z_{0}-\psi^{0}\right| \leq \frac{\varepsilon}{8} & & \text { if } j=0 \\
|e(t, j)| & =0 & & \text { if } j=J,
\end{aligned}
$$

and on every nontrivial segment $\left[t_{j}, t_{j+1}\right] \times\{j\}$

$$
\begin{aligned}
|e(t, j)| & =\left|e_{j}^{\prime}(t)\right|=\left|e_{j}(t)\right| \leq \frac{\varepsilon}{4} \quad \forall t \in\left[t_{j}, t_{j+1}\right), \\
|e(t, j)| & =\left|z_{j}-\varphi_{j}(t)\right| \leq\left|z_{j}-\psi(t, j)\right|+\left|\psi(t, j)-\varphi_{j}(t)\right| \leq \frac{\varepsilon}{2}
\end{aligned}
$$

for all $(t, j)=\left(t_{j+1}, j\right)$ if $j<J$, and $|e(t, j)|=0$ if $j=J$.

Corollary 4.4. Under Assumption 2.5, if a hybrid arc is a hybrid Krasovskii solution to $\mathcal{H}$ then it is also a hybrid Hermes solution to $\mathcal{H}$.

Proof. Let $\psi: \operatorname{dom} \psi \rightarrow O$ be a hybrid Krasovskii solution to $\mathcal{H}$. Pick $(T, J) \in \operatorname{dom} \psi$ (when dom $\psi$ is compact, it is enough to consider $(T, J)=\sup \operatorname{dom} \psi)$. Let $\psi^{\prime}$ be the truncation of $\psi$ to $\operatorname{dom} \psi^{\prime}=\operatorname{dom} \psi \cap$ $([0, T] \times\{0,1, \ldots, J\})$. By Theorem 4.3, there exist $e_{i}: \operatorname{dom} \psi^{\prime} \rightarrow \mathbb{R}^{n}$ and $\varphi_{i}: \operatorname{dom} \psi^{\prime} \rightarrow O$ such that $\sup _{(t, j) \in \operatorname{dom} e_{i}}\left|e_{i}(t, j)\right| \leq \frac{1}{i}$ and which satisfy conditions (V1) and (V2) of Theorem 4.3 (with $e$ replaced by $e_{i}$ and $\varphi$ replaced by $\left.\varphi_{i}\right)$ and also $\left|\psi(t, j)-\varphi_{i}(t, j)\right| \leq 1 / i$ for every $(t, j) \in \operatorname{dom} \varphi_{i}$. The last bound implies that $\varphi_{i}$ converge graphically to $\psi^{\prime}$. Since $\sup _{(t, j) \in \operatorname{dom} e_{i}}\left|e_{i}(t, j)\right| \leq \frac{1}{i}, \psi$ is a hybrid Hermes solution to $\mathcal{H}$.

\subsection{Krasovskii solutions are control-Hermes solutions}

Below, we consider $f$ and $g$ given as in (5) and we impose Assumption 2.10. The result below is a corollary of Theorem 4.2.

Corollary 4.5. Let $f$ satisfy Assumption 2.10 , and let $\psi:[0, T] \rightarrow \mathbb{R}^{n}$ be an absolutely continuous function that satisfies, for almost all $t \in[0, T], \psi(t) \in \widehat{C}, \dot{\psi}(t)=f(\psi(t))$. Let $\varepsilon>0$ be small enough so that rge $\psi+2 \varepsilon \mathbb{B} \subset O$ and let $L$ be a Lipschitz constant for $f(\cdot, u)$ on $\operatorname{rge} \psi+2 \varepsilon \mathbb{B} \subset O$ for any $u \in \kappa_{c}(\operatorname{rge} \psi+2 \varepsilon \mathbb{B})$. Then, for every $\varphi_{0}$ such that $\left|\varphi_{0}-\psi(0)\right|<\varepsilon \mathrm{e}^{-L T}$, there exists a measurable function $e:[0, T] \rightarrow \mathbb{R}^{n}$ and an absolutely continuous function $\varphi:[0, T] \rightarrow O, \varphi(0)=\varphi_{0}$, such that $\sup _{t \in[0, T]}|e(t)| \leq \varepsilon$,

$$
\varphi(t)+e(t) \in C, \quad \text { for all } t \in[0, T], \quad \dot{\varphi}(t)=f^{\prime}\left(\varphi(t), \kappa_{c}(\varphi(t)+e(t))\right) \text { for almost all } t \in[0, T],
$$

and $|\varphi(t)-\psi(t)| \leq \varepsilon$ for all $t \in[0, T]$.

Proof. Let $\varepsilon>0$ be as assumed, and let $\varepsilon^{\prime}=\varepsilon \mathrm{e}^{-L T}<\varepsilon$. By Theorem 4.2, there exists an absolutely continuous function $z:[0, T] \rightarrow O$ with $z(0)=\varphi_{0}$ and a measurable function $e^{\prime}:[0, T] \rightarrow \mathbb{R}^{n}$ such that

$$
\dot{z}(t)=f^{\prime}\left(z(t)+e^{\prime}(t), \kappa_{c}\left(z(t)+e^{\prime}(t)\right)\right) \text { for almost all } t \in[0, T],
$$

and $|z(t)-\psi(t)| \leq \varepsilon^{\prime},\left|e^{\prime}(t)\right| \leq \varepsilon^{\prime}$, and $z(t)+e^{\prime}(t) \in C$ for all $t \in[0, T]$. Note that $\left|\left(z(t)+e^{\prime}(t)\right)-\psi(t)\right| \leq 2 \varepsilon^{\prime}$. Furthermore, $z+e^{\prime}$ is piecewise constant on $[0, T]$, so $\kappa_{c}\left(z(t)+e^{\prime}(t)\right)$ is measurable, and so the mapping $(x, t) \mapsto f^{\prime}\left(x, \kappa_{c}\left(z(t)+e^{\prime}(t)\right)\right)$ is a Carathéodory mapping: continuous in $x$ for a fixed $t$ and measurable in $t$ for a fixed $x$. Consequently, there exists a solution $\varphi:[0, T] \rightarrow O$ to

$$
\dot{\varphi}(t)=f^{\prime}\left(\varphi(t), \kappa_{c}\left(z(t)+e^{\prime}(t)\right)\right) \text { a.e. and } \varphi(0)=\varphi_{0}
$$

for which $\varphi(0)=z(0)$,

$$
|\dot{\varphi}(t)-\dot{z}(t)| \leq L\left|\varphi(t)-z(t)-e^{\prime}(t)\right| \leq L|\varphi(t)-z(t)|+L \varepsilon^{\prime}
$$


and thus $|\varphi(t)-z(t)| \leq \varepsilon^{\prime}\left(\mathrm{e}^{L T}-1\right)$ for all $t \in[0, T]$. Consequently, for all $t \in[0, T]$,

$$
|\varphi(t)-\psi(t)| \leq|\varphi(t)-z(t)|+|z(t)-\psi(t)| \leq \varepsilon^{\prime} \mathrm{e}^{L T}=\varepsilon
$$

Let $e(t):=z(t)+e^{\prime}(t)-\varphi(t)$, so that $z(t)+e^{\prime}(t)=\varphi(t)+e(t)$. Then, since $z(t)+e^{\prime}(t) \in C$, we have $\varphi(t)+e(t) \in C$ for all $t \in[0, T]$. Furthermore, $\dot{\varphi}(t)=f\left(\varphi(t), \kappa_{c}(\varphi(t)+e(t))\right)$. Finally,

$$
|e(t)| \leq|\varphi(t)-z(t)|+\left|e^{\prime}(t)\right| \leq \varepsilon^{\prime}\left(\mathrm{e}^{L T}-1\right)+\varepsilon^{\prime}=\varepsilon^{\prime} \mathrm{e}^{L T}=\varepsilon .
$$

The next result combines Corollary 4.5 above, and the ideas of Theorem 4.3.

Corollary 4.6. Let Assumption 2.10 hold, and let $\psi: \operatorname{dom} \psi \rightarrow O$ be a hybrid Krasovskii solution to $\mathcal{H}=$ $(f, g, C, D, O)$ with $f$ and $g$ as given in (5). Pick any $\varepsilon>0$ and any $(T, J) \in \operatorname{dom} \psi$. Let $E=\operatorname{dom} \psi \cap([0, T] \times$ $\{0,1, \ldots, J\})$ and express $E$ as $\bigcup_{j=0}^{J}\left(\left[t_{j}, t_{j+1}\right] \times\{j\}\right)$. Then, there exist a measurable function $e: \operatorname{dom} e \rightarrow \mathbb{R}^{n}$, $\operatorname{dom} e=E$ and a hybrid arc $\varphi: \operatorname{dom} \varphi \rightarrow O, \varphi(0,0)=\psi(0,0), \operatorname{dom} \varphi=E$ such that $\sup _{(t, j) \in \operatorname{dom} e}|e(t, j)| \leq \varepsilon$ and

(V1') for all $j=0,1, \ldots, J$,

$\varphi(t, j)+e(t, j) \in C$ for all $t \in\left[t_{j}, t_{j+1}\right), \quad \dot{\varphi}(t, j)=f^{\prime}\left(\varphi(t, j), \kappa_{c}(\varphi(t, j)+e(t, j))\right)$ for almost all $t \in\left[t_{j}, t_{j+1}\right]$;

(V2') for all $j=0,1, \ldots, J-1$,

$$
\varphi\left(t_{j+1}, j\right)+e\left(t_{j+1}, j\right) \in D, \quad \varphi\left(t_{j+1}, j+1\right)=g^{\prime}\left(\varphi\left(t_{j+1}, j\right), \kappa_{d}(\varphi(t, j)+e(t, j))\right)
$$

and $|\varphi(t, j)-\psi(t, j)| \leq \varepsilon$ for every $(t, j) \in \operatorname{dom} \varphi$.

Proof. Let $S:=\operatorname{dom} \psi \cap([0, T] \times\{0,1, \ldots, J\})$, fix $\varepsilon>0$ so that $\psi(t, j)+2 \varepsilon \mathbb{B} \subset O$ for all $(t, j) \in S$, and let $L$ be a Lipschitz constant for $f(\cdot, u)$ on $\{\psi(t, j)+2 \varepsilon \mathbb{B} \mid(t, j) \in S\}$. Note that for any $\delta>0$, at each jump of $\psi$ on $S$, i.e. at each point $(t, j) \in S$ such that there exists $(t, j+1) \in S$, we can find $z_{j} \in D$ arbitrarily close to $\psi(t, j)$ that satisfies $\left|g\left(z_{j}, \kappa_{d}\left(z_{j}\right)\right)-\psi(t, j+1)\right| \leq \delta / 2$. This was already justified in the proof of Theorem 4.3. Since $g$ is continuous in the first variable, locally uniformly in the second variable varying over compact sets, we can pick $z_{j}$ so that $\left|g\left(\psi(t, j), \kappa_{d}\left(z_{j}\right)\right)-\psi(t, j+1)\right| \leq \delta$. Thus, by using $\delta=\min \left\{\frac{\varepsilon}{8}, \frac{\varepsilon}{4} \mathrm{e}^{-L T}\right\}$, for every jump of $\psi$ we obtain points $z_{j} \in D$ such that $\left|z_{j}-\psi(t, j)\right| \leq \frac{\varepsilon}{8}$ and $\left|g\left(\psi(t, j), \kappa_{d}\left(z_{j}\right)\right)-\psi(t, j+1)\right| \leq \min \left\{\frac{\varepsilon}{8}, \frac{\varepsilon}{4} \mathrm{e}^{-L T}\right\}$.

On each segment $\left[t_{j}, t_{j+1}\right] \times\{j\}:=S \cap\left(\mathbb{R}_{\geq 0} \times\{j\}\right) \subset S$ that is nontrivial, i.e. segments with $t_{j+1}>t_{j}$, by Corollary 4.5, there exists an absolutely continuous function $\varphi_{j}:\left[t_{j}, t_{j+1}\right] \rightarrow O$ starting at $\varphi_{j}^{0} \in \psi\left(t_{j}, j\right)+$ $\min \left\{\frac{\varepsilon}{8}, \frac{\varepsilon}{4} \mathrm{e}^{-L T}\right\} \mathbb{B}$, where $\varphi_{j}^{0}=g\left(\psi\left(t_{j}, j\right), \kappa_{d}\left(z_{j-1}\right)\right)$ if $j>0, \varphi_{j}^{0}=\psi^{0}$ if $j=0$, and a measurable function $e_{j}:\left[t_{j}, t_{j+1}\right] \rightarrow \mathbb{R}^{n}$ with $\sup _{t \in\left[t_{j}, t_{j+1}\right]}\left|e_{j}(t)\right| \leq \frac{\varepsilon}{4}$ satisfying

$$
\begin{aligned}
\varphi_{j}(t)+e_{j}(t) \in C, \dot{\varphi}_{j}(t)=f^{\prime}\left(\varphi_{j}(t)+e_{j}(t), \kappa_{c}(\varphi(t)+e(t))\right) & \text { for almost all } t \in\left[t_{j}, t_{j+1}\right] \\
\left|\varphi_{j}(t)-\psi(t, j)\right| \leq \frac{\varepsilon}{4} & \text { for all } t \in\left[t_{j}, t_{j+1}\right] .
\end{aligned}
$$

The rest of the proof is analogous to that of Theorem 4.3.

With Corollary 4.6 at hand, the result below can be shown exactly in the same fashion as Corollary 4.4.

Corollary 4.7. Under Assumption 2.10, if a hybrid arc is a hybrid Krasovskii solution to $\mathcal{H}$ then it is also a hybrid control-Hermes solution to $\mathcal{H}$. 


\section{Hermes and CONTROl-Hermes solutions are Krasovski solutions}

Let $\rho: O \rightarrow(0, \infty)$ be an admissible radius of perturbation, that is, a continuous function such that for all $x \in O, x+\rho(x) \mathbb{B} \subset O$. For each $\delta \in(0,1)$, let $\widehat{\mathcal{H}}^{\delta}$ denote the hybrid system defined by sets

$$
\widehat{C}^{\delta}:=\{x \in O \mid x+\delta \rho(x) \mathbb{B} \cap \widehat{C} \neq \emptyset\}, \quad \widehat{D}^{\delta}:=\{x \in O \mid x+\delta \rho(x) \mathbb{B} \cap \widehat{D} \neq \emptyset\}
$$

and mappings

$$
\begin{gathered}
\widehat{f}^{\delta}(x):=\operatorname{co} \widehat{f}((x+\delta \rho(x) \mathbb{B}) \cap C)+\delta \rho(x) \mathbb{B}, \\
\widehat{g}^{\delta}(x):=\{\xi+\delta \rho(\xi) \mathbb{B} \mid \xi \in \widehat{g}((x+\delta \rho(x) \mathbb{B}) \cap D)\} .
\end{gathered}
$$

Such "inflations" of the hybrid system $\widehat{\mathcal{H}}$ were discussed in detail in [23]. A key property of such perturbations of $\widehat{\mathcal{H}}$ is that, thanks to properties of $\widehat{\mathcal{H}}$ listed in Theorem 2.17 and subject to minor local boundedness conditions, a sequence of solutions to $\widehat{\mathcal{H}}^{\delta}$ with decreasing $\delta$ is a solution to $\widehat{\mathcal{H}}$. We will use this below.

Proposition 5.1. Under Assumption 2.10, if a hybrid arc is a hybrid control-Hermes solution to $\mathcal{H}$, then it is also a hybrid Krasovskii solution to $\mathcal{H}$.

Proof. Let $\varphi: \operatorname{dom} \varphi \rightarrow O$ be a hybrid control-Hermes solution to $\mathcal{H}=(f, g, C, D, O)$. Pick $(T, J) \in \operatorname{dom} \varphi$ and let $\varphi^{\prime}$ be the truncation of $\varphi$ to $\operatorname{dom} \varphi^{\prime}$ given by $\operatorname{dom} \varphi \cap([0, T] \times\{0,1, \ldots, J\})$. Let the sequences of $\varphi_{i}$ 's, $e_{i}$ 's, and $\varepsilon_{i}$ 's correspond to $\varphi^{\prime}$ and $(T, J) \in \operatorname{dom} \varphi^{\prime}$, as described in Definition 2.7. In particular, for all $i$ and all $(t, j) \in \operatorname{dom} e_{i},\left|e_{i}(t, j)\right| \leq \varepsilon_{i}$ and $\lim _{i \rightarrow \infty} \varepsilon_{i}=0$.

For each $j \in\{0,1, \ldots, J\}, \varphi_{i}(\cdot, j)$ converge graphically to $\varphi^{\prime}(\cdot, j)$; this comes directly from the definition of graphical convergence. Since, for each $j$, the graphs of $\varphi_{i}(\cdot, j)$ are connected and the graph of $\varphi^{\prime}(\cdot, j)$ is compact (in fact a compact subset of $\mathbb{R}_{\geq 0} \times \mathbb{N} \times O$ ), for each $j$ the graphs of $\varphi_{i}(\cdot, j)$ are eventually bounded with respect to $O$. This can be shown in the same fashion as [39], Corollary 4.12. Consequently, there exists a compact set $K \subset O$ and an index $i^{0}$ such that $\varphi_{i}(t, j) \in K$ for all $i>i^{0}$, all $(t, j) \in \operatorname{dom} \varphi_{i}$.

Let $\rho$ be any admissible perturbation radius. Then let $i_{k}>i_{0}$ be such that $\left|\varepsilon_{i_{k}}\right| \leq k^{-1} \min \{\rho(x) \mid x \in K\}$, and write $\psi_{k}$ to denote $\varphi_{i_{k}}$ to $\operatorname{dom} \psi_{i_{k}}:=\left\{(t, j) \in \operatorname{dom} \varphi_{i_{k}} \mid j \leq J\right\}$. Fix $k$. For all $j$ and almost all $t$ such that $(t, j) \in \operatorname{dom} \psi_{k}$ we have $\psi_{k}(t, j)+e_{i_{k}}(t, j) \in C \subset \widehat{C}$ and since $\left|e_{i_{k}}(t, j)\right| \leq k^{-1} \rho\left(\psi_{k}(t, j)\right)$, we have

$$
\left(\psi_{k}(t, j)+k^{-1} \rho\left(\psi_{k}(t, j)\right)\right) \cap \widehat{C} \neq \emptyset
$$

and consequently $\psi_{k}(t, j) \in \widehat{C}^{1 / k}$. Furthermore, since for any $\delta \in(0,1)$ and any $x$,

$$
f^{\prime}\left(x, \kappa_{c}((x+\delta \rho(x) \mathbb{B}) \cap C)\right) \subset f((x+\delta \rho(x) \mathbb{B}) \cap C) \subset \widehat{f}((x+\delta \rho(x) \mathbb{B}) \cap C) \subset \widehat{f}^{\delta}(x),
$$

we have, for all $j$ and almost all $t$ such that $(t, j) \in \operatorname{dom} \psi_{k}, \dot{\psi}_{k}(t, j) \in \widehat{f}^{1 / k}\left(\psi_{k}(t, j)\right)$. Similar arguments show that for all $(t, j) \in \operatorname{dom} \psi_{k}$ such that $(t, j+1) \in \operatorname{dom} \psi_{k}$ we have $\psi_{k}(t, j) \in \widehat{D}^{1 / k}$ and $\psi_{k}(t, j+1) \in \widehat{g}^{1 / k}\left(\psi_{k}(t, j)\right)$. Consequently, $\psi_{k}$ is a solution to $\widehat{\mathcal{H}}^{1 / k}$, for $k=1,2, \ldots$ Since the sequence of $\varphi_{i}$ 's converges graphically to $\varphi^{\prime}$, by the very definition of graphical convergence, so does the sequence of $\psi_{k}$ 's. Now, since $\left\{\psi_{k}\right\}_{k=1}^{\infty}$ is eventually bounded, its graphical limit, that is $\varphi^{\prime}$, is a solution to $\mathcal{H}$; see [23], Theorems 5.1 and 5.4. Thus, $\varphi^{\prime}$ is a Krasovskii solution to $\mathcal{H}$. Since $(T, J)$ was an arbitrary point in $\operatorname{dom} \varphi$, this is sufficient to guarantee that $\varphi$ is a Krasovskii solution to $\mathcal{H}$.

In the proof above, the local boundedness and continuity properties from Assumption 2.10 are only needed to guarantee that $f$ and $g$ meet Assumption 2.5, so that [23], Theorems 5.1 and 5.4, can be invoked. Furthermore, the proof can be repeated with little change for the case of a set valued $g$, as considered for Hermes solutions. The results of [23] still apply, and thus we obtain:

Corollary 5.2. Under Assumption 2.5, if a hybrid arc is a hybrid Hermes solution to $\mathcal{H}$, then it is also a hybrid Krasovskii solution to $\mathcal{H}$. 
Remark 5.3. To be consistent with what is encountered in hybrid feedback control design, we did not allow $\kappa_{d}$, in the definition of hybrid Hermes-control solutions, to be set-valued. However, the results above, and the results of Section 4.2 can be easily adjusted to account for a set-valued $\kappa_{d}$. In particular, a "set-valued $g$ " version of Corollary 4.6 can be shown like Theorem 4.3. In summary, Theorem 3.2 holds if Assumption 2.10 is altered to say that $\kappa_{d}: O \rightrightarrows \mathbb{R}^{m_{d}}$ is a locally bounded set-valued mapping.

Remark 5.4. We want to point out that similar results, about the equivalence of hybrid Krasovskii and hybrid Hermes concepts of a solution, are true if one seemingly makes the definition of hybrid Hermes solutions broader, by requiring, in (H1) of Definition 2.7, that $\varphi_{i}(t, j)+e_{i}(t, j) \in C$ for almost all $t \in\left[t_{j}^{i}, t_{j+1}^{i}\right]$. This condition is met by $\varphi_{i}$ 's in the original hybrid Hermes solution definition, and so each hybrid Krasovskii solution is a hybrid Hermes solution understood with the above mentioned change. For the other direction, the proof of Proposition 5.1 above still applies.

However, if one alters the definition of hybrid Hermes solutions to be like CADLAG solutions then such hybrid Hermes solutions are still hybrid Krasovskii solutions, but not vice versa. More specifically, suppose that we change Definition 2.7 and consider graphical limits as in Definition 2.7 but with $\varphi_{i}: \bigcup_{j=0}^{J}\left(\left[t_{j}^{i}, t_{j+1}^{i}\right) \times\{j\}\right) \rightarrow O$ and $e_{i}: \bigcup_{j=0}^{J}\left(\left[t_{j}^{i}, t_{j+1}^{i}\right) \times\{j\}\right) \rightarrow \mathbb{R}^{n}$ and with (H1), (H2) replaced by (H1'), (H2'), respectively, that are given by

(H1') for all $j=0,1, \ldots, J$, $\varphi_{i}(t, j)+e_{i}(t, j) \in C$ for all $t \in\left[t_{j}^{i}, t_{j+1}^{i}\right), \quad \dot{\varphi}_{i}(t, j)=f\left(\varphi_{i}(t, j)+e_{i}(t, j)\right)$ for almost all $t \in\left[t_{j}^{i}, t_{j+1}^{i}\right) ;$

(H2') for all $j=0,1, \ldots, J-1$,

$$
\lim _{t \nearrow t_{j+1}^{i}}\left(\varphi_{i}(t, j)+e_{i}(t, j)\right) \in D, \quad \varphi\left(t_{j+1}^{i}, j+1\right)=g\left(\lim _{t \nearrow t_{j+1}^{i}}\left(\varphi_{i}(t, j)+e_{i}(t, j)\right)\right) .
$$

One can extend such $\varphi_{i}$ and $e_{i}$ to the hybrid time domain $\bigcup_{j=0}^{J}\left(\left[t_{j}^{i}, t_{j+1}^{i}\right] \times\{j\}\right)$ by considering mappings whose graphs are exactly the closures of the graphs of $\varphi_{i}$ and $e_{i}$. (This is equivalent to what was suggested in Sect. 2.2.) Such obtained "closures" of $\varphi_{i}$ and $e_{i}$ meet the conditions of Definition 2.7, and their graphical limit is the same as that of $\varphi_{i}$ and $e_{i}$; see [39], Proposition 4.4. Consequently, each hybrid Hermes solution in the sense of the altered definition given above are hybrid Krasovskii solutions.

The opposite direction, however, fails. Indeed, consider a hybrid system on $\mathbb{R}^{2}$ with $C=\left\{x \in \mathbb{R}^{2} \mid x_{2}<0\right\}$, $D=\left\{x \in \mathbb{R}^{2} \mid x_{2}>0\right\}, f(x)=\left(\begin{array}{l}1 \\ 0\end{array}\right)$, and with $g(x)$ being any function on $\mathbb{R}^{2}$. Then a hybrid arc $\psi$ on the hybrid time domain $([0,1] \times\{0\}) \cup([1,1] \times\{1\})$ given by $\psi(t, 0)=(t, 0)$ for all $t \in[0,1], \psi(1,1)=g(\psi(1,0))$ is a hybrid Krasovskii solution. Indeed, $\widehat{C}=\left\{x \in \mathbb{R}^{2} \mid x_{2} \leq 0\right\}, \widehat{D}=\left\{x \in \mathbb{R}^{2} \mid x_{2} \geq 0\right\}, \widehat{f}(x)=f(x), g(x) \in \widehat{g}(x)$ for all $x \in \widehat{D}$, and thus $\psi(t, 0) \in \widehat{C}$ for $t \in[0,1]$ and $\psi(1,0) \in \widehat{D}$. However, $\psi$ just described does not meet the conditions of the altered definition of hybrid Hermes solutions. Indeed, for any $\tau>0$, if $\varphi_{i}(t, 0)+e_{i}(t, 0) \in C$ for all $t \in[0, \tau)$, then $\lim _{t \nearrow \tau}\left(\varphi_{i}(t, 0)+e_{i}(t, 0)\right) \in \widehat{C}$ but $\lim _{t / \tau}\left(\varphi_{i}(t, 0)+e_{i}(t, 0)\right) \notin D$. Hence $\varphi_{i}$ will never jump, and so a graphical limit of such $\varphi_{i}$ 's will never jump.

The described failure is due to the fact that the limiting condition in (H2') places a continuity condition on the measurement noise at the jump times. This may not be reasonable, and one could instead talk about $\varphi_{i}: \bigcup_{j=0}^{J}\left(\left[t_{j}^{i}, t_{j+1}^{i}\right) \times\{j\}\right) \rightarrow O$ but $e_{i}: \bigcup_{j=0}^{J}\left(\left[t_{j}^{i}, t_{j+1}^{i}\right] \times\{j\}\right) \rightarrow \mathbb{R}^{n}$ and replace (H2') by

(H2") for all $j=0,1, \ldots, J-1$,

$$
\lim _{t \nearrow t_{j+1}^{i}} \varphi_{i}(t, j)+e_{i}\left(t_{j+1}^{i}, j\right) \in D, \quad \varphi\left(t_{j+1}^{i}, j+1\right)=g\left(\lim _{t \nearrow t_{j+1}^{i}} \varphi_{i}(t, j)+e_{i}\left(t_{j+1}, j\right)\right) .
$$

It is immediate that such concept of hybrid Hermes solutions is equivalent to hybrid Krasovskii solutions. 


\section{EXAmples}

Here we discuss examples that illustrate that generalized solutions to hybrid systems play a very important role in the robust stabilization problem.

Example 6.1 (impulsive and reset control systems). State-dependent impulsive systems are dynamical systems with states that jump when a condition of the state is satisfied, and flow otherwise. One of the earliest references is the work by Bainov and Simeonov [5]. State-dependent impulsive systems are generally modeled as [14]

$$
\begin{aligned}
\dot{x} & =f(x):=f_{c}(x) & & x \notin \mathcal{M} \\
x^{+} & =g(x):=x+f_{d}(x) & & x \in \mathcal{M}
\end{aligned}
$$

where the function $f_{c}$ defines the continuous dynamics, the function $f_{d}$ defines the discrete dynamics, and $\mathcal{M}$ is the reset set. In most applications, the reset set $\mathcal{M}$ defines a surface in $\mathbb{R}^{n}$ (see e.g. the modeling examples in [14] and the feedback control strategies proposed in [40,43]). A particular case of state-dependent impulsive system is reset control systems, see e.g. $[6,16,32,49]$. A reset controller is a linear system with the property that its output is reset to zero whenever its input and output satisfy certain algebraic condition. (The first reset integrator was introduced in [16] in order to improve the performance of linear systems.) Among several models for reset control systems, the following model has been widely used [6]

$$
\begin{aligned}
\dot{x} & =f(x):=A_{c l} x+B_{c l} d & & x \notin \mathcal{M} \\
x^{+} & =g(x):=A_{R} x & & x \in \mathcal{M}
\end{aligned}
$$

where $\mathcal{M}:=\left\{x \in \mathbb{R}^{n} \mid C_{c l} x=0,\left(I-A_{R}\right) x \neq 0\right\} ; A_{c l}, B_{c l}, C_{c l}$ are the closed-loop system matrices; $A_{R}$ is the reset control matrix; $x$ is the state of the system; and $d$ is an exogenous signal.

State-dependent impulsive systems, and in particular, reset control systems, are hybrid systems that can be modeled with data $(f, g, C, D)$ where the jump set is given by $D:=\mathcal{M}$ and the flow set is given by $C:=\mathbb{R}^{n} \backslash D$. It follows from the definition of $\mathcal{M}$ that the flow set corresponds to almost every point in the state space. Given a state-dependent impulsive system $\mathcal{H}$, its Krasovskii regularization $\widehat{\mathcal{H}}=(\widehat{f}, \widehat{g}, \widehat{C}, \widehat{D}, O)$ with $O=\mathbb{R}^{n}$ has $\widehat{f} \equiv f, \widehat{g} \equiv g, \widehat{C}=\mathbb{R}^{n}$, and $\widehat{D}=D$. Since the flow set is the entire state space, there exist Krasovskii solutions to $\mathcal{H}$ that never jump. It follows by Theorem 3.1 that there exist Hermes solutions to $\mathcal{H}$ that never jump. In fact, it is easy to construct a convergent sequence of solutions to $\mathcal{H}$ with vanishing measurement noise that never hits $D$. Such a limiting solution, a Hermes solution to $\mathcal{H}$, is indeed captured in the Krasovskii regularization of $\mathcal{H}$ above. (See also Ex. 2.18 where for a simpler state-dependent impulsive control system we highlighted the same undesired behavior.)

Generally, the reset control system in (9)-(10) is implemented and simulated with a zero-cross detection (ZCD) algorithm that does not miss the jumps in the presence of noise (for example, in Simulink, one usually uses special blocks like "Compare to zero", "Hit crossing", etc.). Like in Example 2.19, if the reset control system (9)-(10) is implemented with a ZCD algorithm then the hybrid model changes: the state space is extended and the flow and jump sets are redefined. This change leads to robustness to measurement noise. An alternative approach, which does not involve extending the state space, corresponds to thickening the jump set in order to obtain robustness. (Cf. Ex. 6.2.) In [49], the authors used jump sets formed from sectors for reset control systems. Such jump sets were rationalized from the physics of the Clegg integrator in [16]. They also allowed for significant improvements in Lyapunov conditions for asymptotic stability in reset control systems compared to those given in [6].

Example 6.2 (switching on surfaces). In many robotics applications, navigation algorithms for mobile robots are designed by switching between several feedback laws when the state of the system hits a switching surface; see e.g. $[1,7,20]$. For example, consider the scenario in [7], Section 3, where it is desired to steer a vehicle in the plane from its initial location to a target while avoiding an obstacle. A hysteresis-type switching scheme 


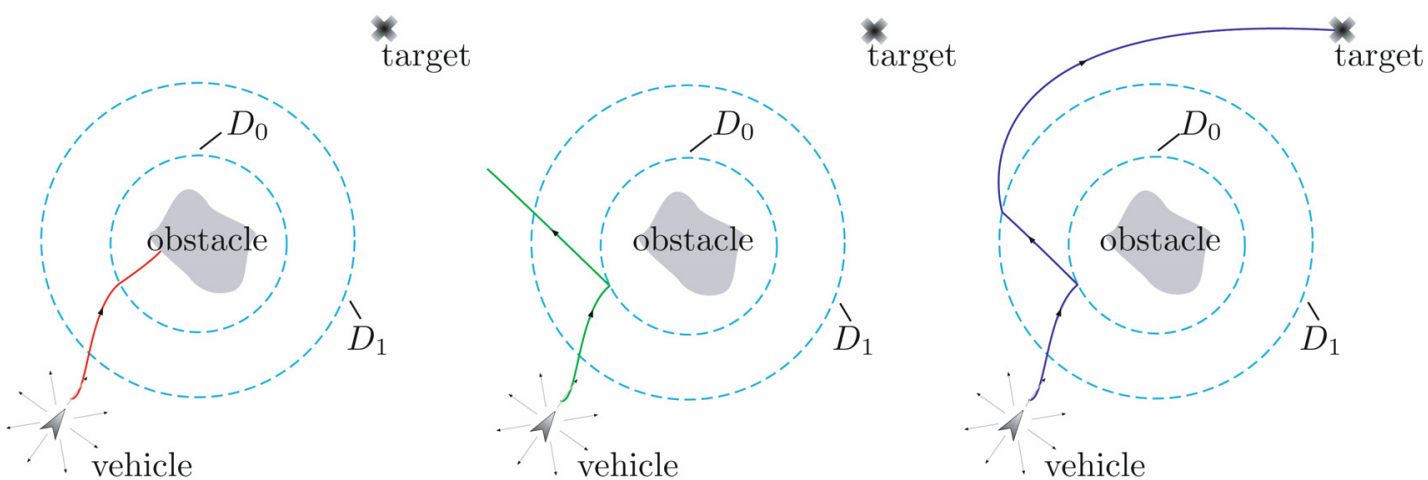

FiguRE 6. Steering a vehicle to its target; a hybrid closed-loop system with logic variables. The circles represent the switching surfaces for the control strategy. The three different controlHermes/Krasovskii solutions in Example 6.2 are depicted: 1) the vehicle crashes into the obstacle (left); 2) the vehicle avoids the obstacle but does not acquire the target (middle); 3) the vehicle avoids the obstacle and acquires the target (right).
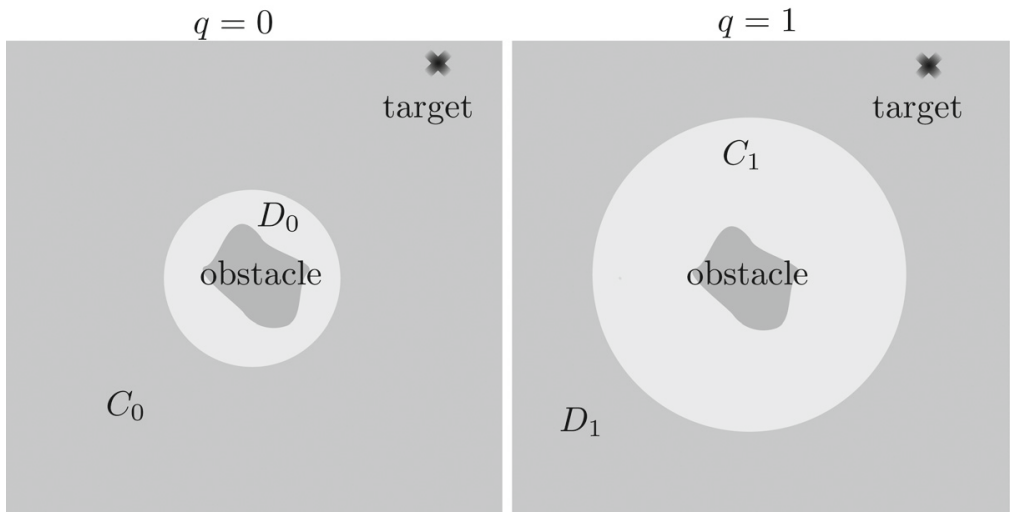

Figure 7. Modified flow and jump sets in Example 6.2 for $q=0$ and $q=1$. The jumps are enforced when the trajectories leave the set $C_{q}$ for the current $q \in\{0,1\}$. Solutions starting away from the obstacle converge to the target even in the presence of small measurement noise.

is proposed in [7]. As depicted in Figure 6, the basic idea is to define two sets given by circles, $D_{0}$ and $D_{1}$, and design control laws, $\kappa_{0}$ and $\kappa_{1}$, so that when $\kappa_{0}$ is applied, the vehicle approaches the target, while when $\kappa_{1}$ is applied, the vehicle is driven away from the obstacle.

Without changing the control strategy described above, we write the closed-loop system as a hybrid system, let $q \in Q:=\{0,1\}$ be a set of modes, $z \in \mathbb{R}^{2}$ the position of the vehicle, and $\dot{z}=f^{\prime}(z, u)$ the dynamics of the vehicle with input $u \in \mathbb{R}^{2}$. Let $x$ be the vector resulting from stacking $z$ and $q$. The closed-loop hybrid system is given by

$$
\begin{aligned}
\dot{x}=f(x):=\left(\begin{array}{c}
f^{\prime}\left(z, \kappa_{q}(z)\right) \\
0
\end{array}\right) & x \notin D_{q} \times\{q\} \\
x^{+}=g(x):=\left(\begin{array}{c}
z \\
1-q
\end{array}\right) & x \in D_{q} \times\{q\} .
\end{aligned}
$$

Let us denote it by $\mathcal{H}=(f, g, C, D)$ where $C:=\cup_{q \in Q}\left(\mathbb{R}^{2} \backslash D_{q}\right) \times\{q\}$, and $D:=\cup_{q \in Q}\left(D_{q} \times\{q\}\right)$. It follows that the Krasovskii regularization of $\mathcal{H}$, denoted by $\widehat{\mathcal{H}}=(\widehat{f}, \widehat{g}, \widehat{C}, \widehat{D}, O)$, has data given by $\widehat{f} \equiv f, \widehat{g} \equiv g$, 
$\widehat{C}=\mathbb{R}^{2} \times Q$, and $\widehat{D}=D$. Since for each $q \in Q$, the set $\widehat{C}$ allows flows for every $z \in \mathbb{R}^{2}$, there exist Krasovskii solutions to $\mathcal{H}$ that never jump. Then Theorem 3.2 and the definition of control-Hermes solutions imply that there exist solutions influenced by arbitrarily small measurement noise that either crash into the obstacle or miss the target. For example, suppose that initially the controller is in mode $q=0$ and consequently, it is driven towards the target. If it gets close to the set $D_{0}$, (arbitrarily small) noise in the measurement of the position of the robot can prevent the controller from detecting that $D_{0}$ was hit and cause the vehicle to crash into the obstacle. A similar argument shows that under (arbitrarily small) measurement noise, the controller can miss the jump at the set $D_{1}$, and thus, causing the vehicle to miss the target. All of the possible controlHermes/Krasovskii solutions, including the one that actually reaches the target with two jumps, are depicted in Figure 6.

Note that the nonrobustness phenomenon in this example is not due to the existence of obstacles itself (see [46]); it is due to the fact that the control strategy switches when the state $z$ belongs to either of the surfaces $D_{0}$ and $D_{1}$, switching condition that is vulnerable to small measurement noise in $z$. A possible modification of $\mathcal{H}$ to accomplish the task robustly is to replace $D_{0}$ by the closed disk that it defines and $D_{1}$ by its (closed) complement, and take $C:=\cup_{q \in Q}\left(C_{q} \times\{q\}\right), C_{q}=\overline{\left(\mathbb{R}^{2} \backslash D_{q}\right)}$ for each $q \in Q$, as shown in Figure 7 . This implementation is an alternative to the zero-crossing detection algorithm used in Example 2.19. It has the features that no extra states are introduced, solutions exist from every initial condition (except those starting on the obstacle), and the data meets the regularity requirements.

\section{Conclusions}

In this paper, motivated by the problem of robust stabilization of hybrid systems, we have discussed the concepts of hybrid Krasovskii, Hermes, and control-Hermes solutions. We have established that the Krasovskii and the Hermes concepts of solutions agree for general hybrid systems, while the Krasovskii and the controlHermes concepts agree for hybrid systems with measurement noise affecting the input or entering through the state-feedback law. This equivalence implies that hybrid Krasovskii solutions can be approximated with arbitrary precision by solutions to the unregularized system with (arbitrarily small) measurement noise. By examples of theoretical and practical relevance, we have motivated the use of generalized solutions in the design of robust hybrid control systems.

\section{REFERENCES}

[1] R.C. Arkin, Behavior Based Robotics. The MIT Press (1998).

[2] J.-P. Aubin and A. Cellina, Differential Inclusions. Springer-Verlag (1984).

[3] J.-P. Aubin, J. Lygeros, M. Quincampoix, S.S. Sastry and N. Seube, Impulse differential inclusions: a viability approach to hybrid systems. IEEE Trans. Aut. Cont. 47 (2002) 2-20.

[4] A. Back, J. Guckenheimer and M. Myers, A dynamical simulation facility for hybrid systems, in Hybrid Systems, Lect. Notes Comput. Sci. 36 (1993) 255-267.

[5] D.D. Bainov and P.S. Simeonov, Systems with Impulse Effect: Stability, Theory, and Applications. Ellis Horwood Limited (1989).

[6] O. Beker, C.V. Hollot, Y. Chait and H. Han, Fundamental properties of reset control systems. Automatica 40 (2004) 905-915.

[7] M. Boccadoro, Y. Wardi, M. Egerstedt and E. Verriest, Optimal control of switching surfaces in hybrid dynamical systems. Discrete Event Dyn. Syst. 15 (2005) 433-448.

[8] M.S. Branicky, Studies in hybrid systems: Modeling, analysis, and control. Ph.D. dissertation, Dept. Elec. Eng. and Computer Sci., MIT (1995).

[9] M.S. Branicky, V.S. Borkar and S.K. Mitter, A unified framework for hybrid control: Model and optimal control theory. IEEE Trans. Aut. Cont. 43 (1998) 31-45.

[10] R.W. Brocket, Hybrid Models for Motion Control Systems, in Essays on Control: Perspectives in the Theory and its Applications, H.L. Trentelman and J.C. Willems Eds., Birkhäuser (1993) 29-53.

[11] M. Broucke and A. Arapostathis, Continuous selections of trajectories of hybrid systems. Systems Control Lett. 47 (2002) 149-157.

[12] C. Cai, A.R. Teel and R. Goebel, Converse Lyapunov theorems and robust asymptotic stability for hybrid systems, in Proc. 24th American Control Conference (2005) 12-17.

[13] F. Ceragioli, Some remarks on stabilization by means of discontinuous feedbacks. Systems Control Lett. 45 (2002) $271-281$. 
[14] V. Chellaboina, S.P. Bhat and W.H. Haddad, An invariance principle for nonlinear hybrid and impulsive dynamical systems. Nonlin. Anal. 53 (2003) 527-550.

[15] F.H. Clarke, Y.S. Ledyaev, E.D. Sontag and A.I. Subbotin, Asymptotic controllability implies feedback stabilization. IEEE Trans. Aut. Cont. 42 (1997) 1394-1407.

[16] J.C. Clegg, A nonlinear integrator for servomechanisms. Transactions A.I.E.E. 77 (Part II) 41-42, 1958.

[17] P. Collins, A trajectory-space approach to hybrid systems, in Proc. 16th MTNS (2004).

[18] P. Collins and J. Lygeros, Computability of finite-time reachable sets for hybrid systems, in Proc. 44th IEEE Conference on Decision Control (2005) 4688-4693.

[19] J.-M. Coron and L. Rosier, A relation between continuous time-varying and discontinuous feedback stabilization. J. Math. Systems Estimation Control 4 (1994) 67-84.

[20] M. Egerstedt, Behavior based robotics using hybrid automata, in Hybrid Systems: Computation and Control, Lect. Notes Comput. Sci. 1790 (2000) 103-116.

[21] A.F. Filippov, Differential equations with discontinuous right-hand sides (English). Matemat. Sbornik. 151 (1960) 99-128.

[22] M. Garavello and B. Piccoli, Hybrid necessary principle. SIAM J. Control Optim. 43 (2005) 1867-1887.

[23] R. Goebel and A.R. Teel, Solutions to hybrid inclusions via set and graphical convergence with stability theory applications. Automatica 42 (2006) 573-587.

[24] R. Goebel, J.P. Hespanha, A.R. Teel, C. Cai and R.G. Sanfelice, Hybrid systems: Generalized solutions and robust stability, in Proc. 6th IFAC Symposium in Nonlinear Control Systems (2004) 1-12.

[25] O. Hàjek, Discontinuous differential equations I. J. Diff. Eqn. 32 (1979) 149-170.

[26] H. Hermes, Discontinuous vector fields and feedback control, in Differential Equations and Dynamical Systems, J.K. Hale and J.P. LaSalle Eds., Academic Press, New York (1967) 155-165.

[27] J.P. Hespanha, Uniform stability of switched linear systems: Extensions of LaSalle's invariance principle. IEEE Trans. Aut. Cont. 49 (2004) 470-482.

[28] J.P. Hespanha, A model for stochastic hybrid systems with application to communication networks. Nonlinear Anal. (Special Issue on Hybrid Systems) 62 (2005) 1353-1383.

[29] C.M. Kellet and A.R. Teel, Smooth Lyapunov functions and robustness of stability for differential inclusions. Systems Control Lett. 52 (2004) 395-405.

[30] N.N. Krasovskii, Game-Theoretic Problems of Capture. Nauka, Moscow (1970).

[31] N.N. Krasovskii and A.I. Subbotin, Game-Theoretical Control Problems. Springer-Verlag (1988).

[32] K.R. Krishnan and I.M. Horowitz, Synthesis of a non-linear feedback system with significant plant-ignorance for prescribed system tolerances. Inter. J. Control 19 (1974) 689-706.

[33] J. Lygeros, K.H. Johansson, S.S. Sastry and M. Egerstedt, On the existence of executions of hybrid automata, in Proc. 41st Conference on Decision and Control (1999) 2249-2254.

[34] J. Lygeros, K.H. Johansson, S.N. Simić, J. Zhang and S.S. Sastry, Dynamical properties of hybrid automata. IEEE Trans. Aut. Cont. 48 (2003) 2-17.

[35] A.N. Michel, L. Wang and B. Hu, Qualitative Theory of Dynamical Systems. Dekker (2001).

[36] D. Nesic, L. Zaccarian and A.R. Teel, Stability properties of reset systems, in Proc. 16th IFAC World Congress in Prague (2005).

[37] C. Prieur, Asymptotic controllability and robust asymptotic stabilizability. SIAM J. Control Optim. 43 (2005) $1888-1912$.

[38] C. Prieur, R. Goebel and A.R. Teel, Results on robust stabilization of asymptotically controllable systems by hybrid feedback, in Proc. 44th IEEE Conference on Decision and Control and European Control Conference (2005) 2598-2603.

[39] R.T. Rockafellar and R.J.-B. Wets, Variational Analysis. Springer (1998).

[40] A.V. Roup, D.S. Bernstein, S.G. Nersesov, W.M. Haddad and V. Chellaboina, Limit cycle analysis of the verge and foliot clock escapement using impulsive differential equations and Poincaré maps. Inter. J. Control 76 (2003) 1685-1698.

[41] R.G. Sanfelice, R. Goebel and A.R. Teel, Results on convergence in hybrid systems via detectability and an invariance principle, in Proc. 24th IEEE American Control Conference (2005) 551-556.

[42] J. Sprinkle, A.D. Ames, A. Pinto, H. Zheng and S.S. Sastry, On the partitioning of syntax and semantics for hybrid systems tools, in Proc. 44th IEEE Conference on Decision and Control and European Control Conference (2005).

[43] S.Y. Tang and R.A. Cheke, State-dependent impulsive models of integrated pest management (IPM) strategies and their dynamic consequences. J. Math. Biol. 50 (2005) 257-292.

[44] L. Tavernini, Differential automata and their discrete simulators. Nonlin. Anal. 11 (1987) 665-683.

[45] L. Tavernini, Generic properties of impulsive hybrid systems. Dynamic Systems \& Applications 13 (2004) 533-551.

[46] S.E. Tuna, R.G. Sanfelice, M.J. Messina and A.R. Teel, Hybrid MPC: Open-minded but not easily swayed, in Proc. International Workshop on Assessment and Future Directions of Nonlinear Model Predictive Control (2005).

[47] A. van der Schaft and H. Schumacher, An Introduction to Hybrid Dynamical Systems, Lecture Notes in Control and Information Sciences. Springer (2000).

[48] H.S. Witsenhausen, A class of hybrid-state continuous-time dynamic systems. IEEE Trans. Aut. Cont. 11 (1966) 161-167.

[49] L. Zaccarian, D. Nesic and A.R. Teel, First order reset elements and the Clegg integrator revisited, in Proc. 24th American Control Conference (2005) 563-568. 